\title{
COMPLEX CUBIC SPLINES
}

\author{
BY \\ J. H. AHLBERG, E. N. NILSON AND J. L. WALSH
}

Introduction. The past two decades have witnessed an increasing intensity of investigation, [1]-[26], into the properties of spline functions. These functions, which in their simplest form yield the analytic counterpart of the draftsman's tool for drawing a smooth curve through a number of prescribed points, play an important and fundamental role in many parts of numerical analysis.

Many of the properties which these functions possess, such as the minimum curvature property [3], [8], [13], [14], are associated with obvious attributes of the elastic beam. Some relate to the best approximation of linear operators [15], [20], and have rather profound meanings in approximation theory. Others, such as orthogonality [24], rate of convergence [12], [17], [23], [25], and completeness [25] of certain bases of splines, are at first rather surprising. A few (cf. [22, p. 241]) are still quite puzzling.

The application of spline theory to the approximation of a function analytic interior to a rectifiable Jordan curve and continuous in the corresponding closed region has been considered to a limited extent in an earlier paper, [24], by the authors. There splines were treated which are piecewise polynomial in the arc length $s$ on the curve. The present development is concerned with cubic splines in the complex variable $z$ and provides some insight into the structure of the spline approximation generally. In particular, it serves to establish a connection with the classical theory of approximation to an analytic function.

As part of this development, proofs are given for the convergence of the complex cubic spline and its derivatives for the situations in which the approximated function is of class $C^{\alpha}(\alpha=0,1,2,3$, or 4$)$ on the boundary. These may be modified in an obvious manner for the standard real cubic spline and for the convergence properties of second and third derivatives. There result noteworthy simplifications over proofs already existing in the literature [12], [17], for $\alpha=2,3,4$.

The convergence properties of cubic splines for cases in which the approximated function is assumed merely to be continuous or to have continuous first derivative constitute significant new developments in spline theory. In addition, a curious spline property is here presented relating to the approximation of the fourth derivative.

The complex spline approximation. Let $K$ be a rectifiable Jordan curve in the complex plane. Let $t_{1}, t_{2}, \ldots, t_{N}$ be points on $K$ arranged in counterclockwise order, separating $K$ into arcs $K_{j}(j=1, \ldots, N)$ with $K_{j}$ the arc from $t_{j-1}$ to $t_{j}\left(t_{N+1}=t_{1}\right)$.

Presented to the Society, November 8, 1965; received by the editors November 15, 1965. 
For this subdivision $\Delta$ of $K$ we form a spline $q_{\Delta}(t)$ on $K$ composed of complex cubics: for $t$ on $K_{j}$,

$$
\begin{aligned}
q_{\Delta}(t)= & \frac{M_{j-1}}{6 h_{j}}\left(t_{j}-t\right)^{3}+\frac{M_{j}}{6 h_{j}}\left(t-t_{j-1}\right)^{3}+\left(\frac{f_{j-1}}{h_{j}}-\frac{M_{j-1} h_{j}}{6}\right)\left(t_{j}-t\right) \\
& +\left(\frac{f_{j}}{h_{j}}-\frac{M_{j} h_{j}}{6}\right)\left(t-t_{j-1}\right),
\end{aligned}
$$

where $h_{j}=t_{j}-t_{j-1}, f_{j}=q_{\Delta}\left(t_{j}\right)$, and $M_{j}=q_{\Delta}^{\prime \prime}\left(t_{j}\right)$. The quantities $M_{j}$ are to be so chosen that the limiting values of $q_{\Delta}^{\prime}\left(t_{j}\right)$, obtained by approach to $t_{j}$ on $K_{j+1}$ and $K_{j}$, are equal: for $j=1,2, \ldots, N$,

$$
\frac{h_{j}}{6} M_{j-1}+\frac{h_{j}+h_{j+1}}{3} M_{j}+\frac{h_{j+1}}{6} M_{j+1}=\frac{f_{j+1}-f_{j}}{h_{j+1}}-\frac{f_{j}-f_{j-1}}{h_{j}}
$$

If we set

we obtain

$$
\lambda_{j}=\frac{h_{j+1}}{h_{j}+h_{j+1}}, \quad \mu_{j}=1-\lambda_{j}
$$

$$
\mu_{j} M_{j-1}+2 M_{j}+\lambda_{j} M_{j+1}=6 f\left[t_{j-1}, t_{j}, t_{j+1}\right] .
$$

Here $f\left[t_{j-1}, t_{j}, t_{j+1}\right]$ is the second divided difference [27] involving functional values $f_{j-1}, f_{j}, f_{j+1}$ at the points $t_{j-1}, t_{j}, t_{j+1}$.

The existence of the spline $q_{\Delta}(t)$ on $K$, assuming prescribed values $f_{j}$ at the mesh locations $t_{j}$, rests upon the possibility of solving the $N$ simultaneous equations (3) for the quantities $M_{j}$. Now the coefficient matrix $A$ has dominant main diagonal provided that, for each $j(j=1,2, \ldots, N)$,

$$
2\left|h_{j}+h_{j+1}\right|>\left|h_{j}\right|+\left|h_{j+1}\right| \text {. }
$$

This condition is equivalent to requiring, for each $j$, that $t_{j}$ lies within the ellipse with foci $t_{j-1}$ and $t_{j+1}$ and with eccentricity $1 / 2$. If $K$ is a smooth Jordan curve (i.e., has a continuously turning tangent), then this condition is satisfied for sufficiently small mesh norm

$$
\|\Delta\|=\max _{1 \leqq j \leqq N}\left|h_{j}\right| .
$$

We assume throughout the remainder of the paper that $K$ is smooth and that $\|\Delta\|$ is sufficiently small that $A$ be nonsingular. The piecewise cubic function $q_{\Delta}(t)$ then exists and is unique for arbitrary complex values $f_{1}, f_{2}, \ldots, f_{N}$.

Alternatively we may represent the spline $q_{\Delta}(t)$ in terms of its first derivative at the mesh points, $m_{j}=q_{\Delta}^{\prime}\left(t_{j}\right)$. On $K_{j}$ we have

$$
\begin{aligned}
q_{\Delta}(t)= & \frac{m_{j-1}}{h_{j}^{2}}\left(t_{j}-t\right)^{2}\left(t-t_{j-1}\right)-\frac{m_{j}}{h_{j}^{2}}\left(t-t_{j-1}\right)^{2}\left(t_{j}-t\right) \\
& +\frac{f_{j-1}}{h_{j}^{3}}\left(t_{j}-t\right)^{2}\left[2\left(t-t_{j-1}\right)+h_{j}\right]+\frac{f_{j}}{h_{j}^{3}}\left(t-t_{j-1}\right)^{2}\left[2\left(t_{j}-t\right)+h_{j}\right],
\end{aligned}
$$


and the quantities $m_{j}$ satisfy the requirement that $q_{\Delta}^{\prime \prime}\left(t_{j}-\right)=q_{\Delta}^{\prime \prime}\left(t_{j}+\right)$ for $j=1$, $2, \ldots, N$ :

$$
\frac{m_{j-1}}{h_{j}}+2\left(\frac{1}{h_{j}}+\frac{1}{h_{j+1}}\right) m_{j}+\frac{m_{j+1}}{h_{j+1}}=3 \frac{f_{j+1}-f_{j}}{h_{j+1}^{2}}+3 \frac{f_{j}-f_{j-1}}{h_{j}^{2}},
$$

which may be rewritten in the form

$$
\lambda_{j} m_{j-1}+2 m_{j}+\mu_{j} m_{j+1}=3\left[\mu_{j} \frac{f_{j+1}-f_{j}}{h_{j+1}}+\lambda_{j} \frac{f_{j}-f_{j-1}}{h_{j}}\right] .
$$

The complex spline $S_{\Delta}(z)$ is now defined interior to $K$ by the Cauchy integral

$$
S_{\Delta}(z)=\frac{1}{2 \pi i} \int_{K} \frac{q_{\Delta}(t) d t}{t-z},
$$

with $S_{\Delta}(t), t$ on $K$, as the limiting value (cf. [29]) for approach from within $K$,

$$
S_{\Delta}(t)=\frac{1}{2} q_{\Delta}(t)+\frac{1}{2 \pi i} \int_{K} \frac{q_{\Delta}(\tau) d \tau}{\tau-t} .
$$

Convergence on the boundary. We consider first the convergence of $\left\{q_{\Delta_{k}}(t)\right\}$ and the corresponding sequences of derivatives for a sequence of meshes $\left\{\Delta_{k}\right\}$ with $\left\|\Delta_{k}\right\| \rightarrow 0$ as $k \rightarrow \infty$. Let the mesh points of $\Delta_{k}$ be $t_{k, 1}, t_{k, 2}, \ldots, t_{k, N_{k}}$. The row-max norm of the inverse of the coefficient matrix $A_{k}$ in (3), or $B_{k}$ in (7) does not exceed (cf. [10, p. 97]) $\left\{\min _{j}\left[2-\left|\lambda_{k, j}\right|-\left|\mu_{k, j}\right|\right]\right\}^{-1}$ and for arbitrary $\eta>0$ this bound can be made not to exceed $1+\eta$ by taking $\left\|\Delta_{k}\right\|$ sufficiently small. Let $b_{k: i, j}^{-1}$ represent the general element of the inverse matrix $B_{k}^{-1}$. For convenience in notation, we will frequently drop the index $k$ on the mesh $\Delta_{k}$ as in the following proof.

Consider the interval $K_{j}$. On $K_{j}$ set $t=\left(t_{j-1}+t_{j}\right) / 2+\varepsilon$. Let $f(t)$ be continuous on $K$. Then from (5) we have

Thus

$$
\begin{aligned}
q_{\Delta}(t)-f(t)= & \left(\frac{1}{2} h_{j}-\varepsilon\right)^{2}\left(\frac{1}{2} h_{j}+\varepsilon\right) \frac{m_{j-1}}{h_{j}^{2}}-\left(\frac{1}{2} h_{j}+\varepsilon\right)^{2}\left(\frac{1}{2} h_{j}-\varepsilon\right) \frac{m_{j}}{h_{j}^{2}} \\
& +\frac{2 f_{j-1}}{h_{j}^{3}}\left(\frac{1}{2} h_{j}-\varepsilon\right)^{2}\left(h_{j}+\varepsilon\right)+\frac{2 f_{j}}{h_{j}^{3}}\left(\frac{1}{2} h_{j}+\varepsilon\right)^{2}\left(h_{j}-\varepsilon\right)-f(t) .
\end{aligned}
$$

$$
\begin{aligned}
\left|q_{\Delta}(t)-f(t)\right| \leqq & \left(\frac{1}{2}\left|h_{j}\right|+\varepsilon\right)^{3} \frac{3(1+\eta) \cdot 2}{\left|h_{j}\right|^{2}} \max _{i}\left[\left|\mu_{i}\right| \frac{\left|f_{i+1}-f_{i}\right|}{\left|h_{i+1}\right|}+\left|\lambda_{i}\right| \frac{\left|f_{i}-f_{i-1}\right|}{\left|h_{i}\right|}\right] \\
& +\left|\frac{f_{j}+f_{j+1}}{2}-f(t)\right|+\left|\frac{3}{2} \frac{\varepsilon}{h_{j}}-\frac{2 \varepsilon^{3}}{h_{j}^{3}}\right|\left|f_{j}-f_{j-1}\right|,
\end{aligned}
$$

since

$$
m_{j}=3 \sum_{j=1}^{N} b_{j, i}^{-1}\left(\mu_{i} \frac{f_{i+1}-f_{i}}{h_{i+1}}+\lambda_{i} \frac{f_{i}-f_{i-1}}{h_{i}}\right)
$$

and $\sum_{k}\left|b_{j, k}^{-1}\right| \leqq 1+\eta$. 
Now $|\varepsilon| /\left|h_{j}\right| \leqq 1 / 2,\left|\mu_{j}\right|<1$, and $\left|\lambda_{j}\right|<1$ for $\|\Delta\|$ sufficiently small. If we assume that the meshes $\Delta$ possess the property that

$$
\left[\max _{j}\|\Delta\| /\left|h_{j}\right|\right] \leqq C_{1}<\infty
$$

and if we designate by $\mu(\delta ; f)$ the modulus of continuity of the function $f(t)$, then on $K_{j}$

$$
\left|q_{\Delta}(t)-f(t)\right| \leqq\left[12(1+\eta) C_{1}+2\right] \mu(\|\Delta\|, f) .
$$

Since the constants are independent of $j$, we have proved

THEOREM 1. Let $f(t)$ be continuous on $K$. Let $\left\{\Delta_{k}\right\}$ be a sequence of subdivisions of $K$ with $\lim _{k \rightarrow \infty}\left\|\Delta_{k}\right\|=0$ and with $\left[\max _{j}\left\|\Delta_{k}\right\| /\left|h_{k, j}\right|\right] \leqq C_{1}<\infty$. Let $q_{\Delta_{k}}(t)$ be the complex cubic spline on K of interpolation to $f(t)$ on $\Delta_{k}$. Then $\left\{q_{\Delta_{k}}(t)\right\} \rightarrow f(t)$ uniformly as $\left\|\Delta_{k}\right\| \rightarrow 0$. If $f(t)$ satisfies a Hölder condition of order $\beta$ on $K(0<\beta \leqq 1)$, then $\left|q_{\Delta_{k}}(t)-f(t)\right|=O\left(\left\|\Delta_{k}\right\|^{\beta}\right)$.

In order to obtain the convergence properties of the complex spline defined in equation (8), it is necessary to show that $q_{\Delta}(t)-f(t)$ or its derivatives satisfy suitable Hölder conditions. These conditions become involved in the definition of the Cauchy Principal Value of the integral in (9).

For $t$ and $\tau$ on $K_{j}$ we obtain from (5) $\left(t^{*}=\left(t_{j-1}+t_{j}\right) / 2\right)$

$$
q_{\Delta}(t)-q_{\Delta}(\tau)
$$

$$
\begin{array}{r}
=(t-\tau)\left\{\left[m_{j-1}+m_{j}-2 \frac{\left.f_{j}-f_{j-1}\right]}{h_{j}}\right]\left[\frac{\left(t-t^{*}\right)^{2}+\left(t-t^{*}\right)\left(\tau-t^{*}\right)+\left(\tau-t^{*}\right)^{2}}{h_{j}^{2}}-\frac{1}{2}\right]\right. \\
\left.+\left(m_{j}-m_{j-1}\right) \frac{t+\tau-2 t^{*}}{2 h_{j}}+\frac{\left(f_{j}-f_{j-1}\right)}{h_{j}}\right\} .
\end{array}
$$

If $f(t)$ satisfies a Hölder condition of $\operatorname{order} \beta(0<\beta \leqq 1)$ and if $0 \leqq \delta \leqq \beta$, it follows when $\|\Delta\|$ is sufficiently small that for $t$ and $\tau$ on $K_{j}$,

$$
\begin{aligned}
& \left|\left[q_{\Delta}(t)-f(t)\right]-\left[q_{\Delta}(\tau)-f(\tau)\right]\right|=|t-\tau|^{\delta}|| \Delta \|^{\beta-\delta} \cdot \frac{|t-\tau|^{\beta-\delta}}{\|\Delta\|^{\beta-\delta}} \\
& \cdot\left\{\left[\left|m_{j-1}+m_{j}\right| \cdot|t-\tau|^{1-\beta}+2 \cdot \frac{\left|f_{j}-f_{j-1}\right|}{\left|h_{j}\right|^{\beta}}\left|\frac{t-\tau}{h_{j}}\right|^{1-\beta}\right] \cdot \frac{1}{4}\right. \\
& \left.+\frac{1}{2}\left|m_{j}-m_{j-1}\right||t-\tau|^{1-\beta}+\left|\frac{t-\tau}{h_{j}}\right|^{1-\beta} \cdot \frac{\left|f_{j}-f_{j-1}\right|}{\left|h_{j}\right|^{\beta}}+\frac{f(\tau)-f(t)}{|\tau-t|^{\beta}}\right\} .
\end{aligned}
$$

Thus, by means of (10), it is seen that $\left[q_{\Delta}(t)-f(t)\right] /\|\Delta\|^{\beta-\delta}$ satisfies a uniform Hölder condition of order $\delta$.

COROLlARY. Under the conditions of Theorem 1 with $f(t)$ satisfying a Hölder condition of order $\beta(0<\beta \leqq 1)$, the function $\left[q_{\Delta_{k}}(t)-f(t)\right] /\left\|\Delta_{k}\right\|^{\beta-\delta}$ satisfies a Hölder condition of order $\delta, 0<\delta \leqq \beta$, uniformly with respect to $k$.

We turn to a consideration of the case in which $f(t)$ has a continuous derivative on $K$. 
THEOREM 2. Let $f(t)$ be of class $C^{1}$ on $K$. Let $\Delta_{k}: t_{k, 1}, t_{k, 2}, \ldots, t_{k, N_{k}}(k=1,2, \ldots)$ represent a sequence of subdivisions of $K$ with $\lim _{k \rightarrow \infty}\left\|\Delta_{k}\right\|=0$. Let $q_{\Delta_{k}}(t)$ be the complex cubic spline on $K$ of interpolation to $f(t)$ at the points of $\Delta_{k}$. Then $\left\{q_{\Delta_{k}}^{\prime}(t)\right\}$ converges uniformly on $K$ to $f^{\prime}(t)$ and $\left[q_{\Delta_{k}}(t)-f(t)\right]=o\left(\left\|\Delta_{k}\right\|\right)$.

Proof. If we write (7) as

$$
B_{k} \mathbf{m}_{k}=3 \mathbf{e}_{k}
$$

where $\mathbf{e}_{k}$ is the $N_{k}$-vector $\left(e_{k, 1}, e_{k, 2}, \ldots, e_{k, N_{k}}\right)^{\top}$ with

$$
e_{k, j}=\mu_{k, j}\left[f\left(t_{k, j+1}\right)-f\left(t_{k, j}\right)\right] / h_{k, j+1}+\lambda_{k, j}\left[f\left(t_{k, j}\right)-f\left(t_{k, j-1}\right)\right] / h_{k, j}
$$

and $\mathbf{m}_{k}$ the corresponding vector of spline first derivatives, $\left(m_{k, 1}, m_{k, 2}, \ldots, m_{k, N_{k}}\right)^{\top}$, then

$$
B_{k}\left(\mathbf{m}_{k}-\mathbf{e}_{k}\right)=\left(3 I_{k}-B_{k}\right) \mathbf{e}_{k} .
$$

Here $I_{k}$ is the $N_{k} \times N_{k}$ identity matrix. The $j$ th row in $3 I_{k}-B_{k}$ has three nonzero elements centered on the main diagonal position: $\lambda_{k, j},-1, \mu_{k, j}$. Thus the right-hand member of (13) has the $j$ th element

$$
\lambda_{k, j} e_{k, j-1}-e_{k, j}+\mu_{k, j} e_{k, j+1}=\lambda_{k, j}\left(e_{k, j-1}-e_{k, j}\right)+\mu_{k, j}\left(e_{k, j+1}-e_{k, j}\right) .
$$

Now $f^{\prime}(t)$ is continuous on $K$ so that $\max _{j}\left|f^{\prime}\left(t_{k, j}\right)-e_{k, j}\right|$ can be made arbitrarily small, uniformly with respect to $k$, by taking $\left\|\Delta_{k}\right\|$ sufficiently small. Thus

$$
\left\|\left(3 I_{k}-B_{k}\right) \mathbf{e}_{k}\right\|=3\left[\max _{j}\left|\lambda_{k, j}\left(e_{k, j-1}-e_{k, j}\right)+\mu_{k, j}\left(e_{k, j+1}-e_{k, j}\right)\right|\right]
$$

can be made uniformly (in $k$ ) arbitrarily small. Hence $\max _{j}\left|m_{k, j}-f^{\prime}\left(t_{k, j}\right)\right| \rightarrow 0$ as $\left\|\Delta_{k}\right\| \rightarrow 0$. For $t$ on $K_{k, j}$ with $t=\left(t_{k, j-1}+t_{k, j}\right) / 2+\varepsilon$, we have

$$
\begin{aligned}
q_{\Delta_{k}}^{\prime}(t) & -\frac{f\left(t_{k, j}\right)-f\left(t_{k, j-1}\right)}{h_{k, j}} \\
& =\left(\frac{3 \varepsilon^{2}}{h_{k, j}^{2}}-\frac{1}{4}\right)\left(m_{k, j-1}+m_{k, j}-2 \frac{f\left(t_{k, j}\right)-f\left(t_{k, j-1}\right)}{h_{k, j}}\right)+\frac{\varepsilon}{h_{k, j}}\left(m_{k, j}-m_{k, j-1}\right) .
\end{aligned}
$$

For $\left\|\Delta_{k}\right\|$ sufficiently small, $\left|\varepsilon / h_{j}\right| \leqq 1$. The uniform convergence of $\left\{q_{\Delta_{k}}^{\prime}(t)\right\}$ to $f^{\prime}(t)$ is a direct consequence. Since for $t$ on $K_{k, j}$ we have

$$
q_{\Delta_{k}}(t)-f(t)=\int_{t_{k, j-1}}^{t}\left[q_{\Delta_{k}}^{\prime}(t)-f^{\prime}(t)\right] d t,
$$

it is evident that $\left[q_{\Delta_{k}}(t)-f(t)\right]=o\left(\left\|\Delta_{k}\right\|\right)$.

Since we may write, for $t$ on $K_{k, j}$,

$$
f^{\prime}(t)-\frac{f\left(t_{k, j}\right)-f\left(t_{k, j-1}\right)}{h_{k, j}}=\frac{1}{h_{k, j}} \int_{t_{k, j-1}}^{t_{k, j}}\left[f^{\prime}(t)-f^{\prime}(\tau)\right] d \tau,
$$


it follows from (14) that $\left[q_{\Delta_{k}}^{\prime}(t)-f^{\prime}(t)\right]=O\left(\left\|\Delta_{k}\right\|^{\beta}\right)$ if $f^{\prime}(t)$ satisfies a Hölder condition of order $\beta$ on $K, 0<\beta \leqq 1$. Thus we have

COROLlaRY 1. Under the conditions of Theorem 2 on $f(t),\left\{\Delta_{k}\right\}$, and $\left\{q_{\Delta_{k}}(t)\right\}$, if $f^{\prime}(t)$ satisfies a Hölder condition of order $\beta$ on $K(0<\beta \leqq 1)$, then for $p=0,1$ we have $f^{(p)}(t)-q_{\Delta_{k}}^{(p)}(t)=O\left(\left\|\Delta_{k}\right\|^{1+\beta-p}\right)$.

We can also prove

COROLlaRY 2. Under the conditions of Corollary $1,\left[q_{\Delta_{k}}^{\prime}(t)-f^{\prime}(t)\right] /\left\|\Delta_{k}\right\|^{\beta-\delta}$ satisfies a uniform Hölder condition of order $\delta, 0 \leqq \delta \leqq \beta$, provided

$$
\left[\max _{j}\left\|\Delta_{k}\right\| /\left|h_{k j}\right|\right] \leqq C_{1}<\infty \text {. }
$$

Proof. We obtain from (5) (dropping the index $k$; again $t^{*}=\left(t_{j-1}+t_{j}\right) / 2$ )

$$
\begin{aligned}
q_{\Delta}^{\prime}(t)-q_{\Delta}^{\prime}(\tau)=(t-\tau)\left\{\left[\frac{m_{j-1}-\left(f,-f_{j-1}\right) / h_{j}}{h_{j}}+\right.\right. & \left.\frac{m_{j}-\left(f_{j}-f_{j-1}\right) / h_{j}}{h_{j}}\right] \\
& \left.\frac{3\left(t+\tau-2 t^{*}\right)}{h_{j}}+\frac{m_{j}-m_{j-1}}{h_{j}}\right\} .
\end{aligned}
$$

It is seen from (13) and the properties of $f^{\prime}(t)$ that the quantities

$$
\frac{m_{j}-\left(f_{j}-f_{j-1}\right) / h_{j}}{\|\Delta\|^{\beta}}, \quad \frac{m_{j-1}-\left(f_{j}-f_{j-1}\right) / h_{j}}{\|\Delta\|^{\beta}}
$$

are uniformly bounded as is $\left(m_{j}-m_{j-1}\right) /\|\Delta\|^{\beta}$. Hence for $\|\Delta\|$ sufficiently small,

$$
\begin{aligned}
\left|\left[q_{\Delta}^{\prime}(t)-f^{\prime}(t)\right]-\left[q_{\Delta}^{\prime}(\tau)-f^{\prime}(\tau)\right]\right| & \\
\leqq|t-\tau|^{\delta}\|\Delta\|^{\beta-\delta} \cdot\left\{\left[3 \frac{\left|m_{j-1}-\left(f_{j}-f_{j-1}\right) / h_{j}\right|}{\|\Delta\|^{\beta}}+3 \frac{\left|m_{j}-\left(f_{j}-f_{j-1}\right) / h_{j}\right|}{\|\Delta\|^{\beta}}\right.\right. & \left.\left.+\frac{\left|m_{j}-m_{j-1}\right|}{\|\Delta\|^{\beta} .}\right]\left|\frac{t-\tau}{h_{j}}\right|^{1-\delta} \cdot \frac{\|\Delta\|^{\delta}}{\left|h_{j}\right|^{\delta}}+\left|\frac{f^{\prime}(\tau)-f^{\prime}(t)}{|\tau-t|^{\beta}}\right|\right\} .
\end{aligned}
$$

The conclusion of the corollary follows.

It is to be noted that the mesh restriction (11) appearing in Theorem 1 is not required in Theorem 2 or in the next theorem. We remark also that by (12) we have, without this mesh restriction,

COROLlARY 3. Under the conditions of Theorem 2, for arbitrary $\delta, 0<\delta \leqq 1$, the quantity $\left[q_{\Delta_{\kappa}}(t)-f(t)\right] /\left\|\Delta_{k}\right\|^{1-\delta}$ satisfies a Hölder condition of order $\delta$ on $K$ uniformly with respect to $k$.

THEOREM 3. Let $f(t),\left\{\Delta_{k}\right\}$, and $\left\{q_{\Delta_{k}}(t)\right\}$ satisfy the requirements of Theorem 2 but with $f(t)$ of class $C^{2}$ on $K$. Then $\left\{q_{\Delta_{k}}^{\prime \prime}(t)\right\} \rightarrow f^{\prime \prime}(t)$ uniformly on $K$ as $k \rightarrow \infty$.

Proof. Write (3) as

$$
A_{k} \mathbf{M}_{k}=3 \mathbf{d}_{k},
$$


where $\mathbf{M}_{k}=\left(M_{k, 1}, M_{k, 2}, \ldots, M_{k, N_{k}}\right)^{\top}$ and $d_{k}$ is the $N_{k}$-vector whose $j$ th component is $2 f\left[t_{k, j-1}, t_{k, j}, t_{k, j+1}\right]$. Then

$$
A_{k}\left(\mathbf{M}_{k}-\mathrm{d}_{k}\right)=\left(3 I_{k}-A_{k}\right) \mathrm{d}_{k} .
$$

The vector $\left(3 I_{k}-A_{k}\right) \mathrm{d}_{k}$ has norm equal to

$$
3 \max _{j}\left|\mu_{k, j}\left(d_{k, j-1}-d_{k, j}\right)+\lambda_{k, j}\left(d_{k, j+1}-d_{k, j}\right)\right| \text {. }
$$

If $f^{\prime \prime}(t)$ is continuous, then $3 d_{k, j}$ (the right-hand member of (3)) can be made uniformly close to $3 f^{\prime \prime}\left(t_{k, j}\right)$ by taking $\left\|\Delta_{k}\right\|$ sufficiently small. In fact, we have from the Taylor theorem with integral remainder,

$$
\begin{aligned}
& f\left[t_{k, j-1}, t_{k, j}, t_{k, j+1}\right]-\frac{1}{2} f^{\prime \prime}\left(t_{k, j}\right) \\
& =\frac{1}{h_{k, j}+h_{k, j+1}}\left\{\frac{1}{h_{k, j+1}} \int_{t_{k, j}}^{t_{k, j+1}}\left(t_{k, j+1}-\tau\right)\left[f^{\prime \prime}(\tau)-f^{\prime \prime}\left(t_{k, j}\right)\right] d \tau\right. \\
& \left.\quad+\frac{1}{h_{k, j}} \int_{t_{k, j-1}}^{t_{k, j}}\left(\tau-t_{k, j-1}\right)\left[f^{\prime \prime}(\tau)-f^{\prime \prime}\left(t_{k, j}\right)\right] d \tau\right\}
\end{aligned}
$$

The left-hand member of (16), therefore, does not exceed in absolute value the quantity

$$
\begin{aligned}
\left.\max _{t \text { on } K_{k, j}}\left|f^{\prime \prime}(t)-f^{\prime \prime}\left(t_{k, j}\right)\right|\right] \cdot \frac{\left|h_{k, j+1}\right|}{\left|h_{k, j}+h_{k, j+1}\right|} \\
+\left[\max _{t \text { on } K_{k, j-1}}\left|f^{\prime \prime}(t)-f^{\prime \prime}\left(t_{k, j}\right)\right|\right] \cdot \frac{\left|h_{k, j}\right|}{\left|h_{k, j}+h_{k, j+1}\right|} \leqq 2 \mu\left(\left|t-t_{k, j}\right|, f^{\prime \prime}\right)
\end{aligned}
$$

for $\left\|\Delta_{k}\right\|$ sufficiently small.

From the boundedness of $\left\|A_{k}^{-1}\right\|$ (uniform with respect to $k$ ) it follows that $\left\|\mathbf{M}_{k}-\mathbf{d}_{k}\right\| \rightarrow 0$ as $k \rightarrow \infty$. The linearity of $q_{\Delta_{k}}^{\prime \prime}(t)$ between junctions $t_{k, j}$ and the uniform continuity of $f^{\prime \prime}(t)$ now give the conclusion of the theorem.

If $f^{\prime \prime}(t)$ satisfies a Hölder condition of order $\beta$ on $K, 0<\beta \leqq 1$, then it follows from (16) that $\left\|\mathbf{M}_{k}-\mathbf{d}_{k}\right\|=O\left(\left\|\Delta_{k}\right\|^{\beta}\right)$. Moreover, for $t$ on $K_{k, j}$ we have

$$
\frac{f(t)-f\left(t_{k, j-1}\right)}{t-t_{k, j-1}}-f^{\prime}\left(t_{k, j-1}\right)=\frac{1}{t-t_{k, j-1}} \int_{t_{k, j-1}}^{t}(t-\tau) f^{\prime \prime}(\tau) d \tau
$$

and so from the interpolation property

$$
q_{\Delta_{k}}^{\prime}\left(t_{k, j-1}\right)-f^{\prime}\left(t_{k, j-1}\right)=\frac{1}{h_{k, j}} \int_{t_{k, j-1}}^{t_{k, J}}\left(t_{k, j}-\tau\right)\left[q_{\Delta_{k}}^{\prime \prime}(\tau)-f^{\prime \prime}(\tau)\right] d \tau .
$$

Thus $\left[q_{\Delta_{k}}^{\prime}\left(t_{k, j}\right)-f^{\prime}\left(t_{k, j}\right)\right]=O\left(\left\|\Delta_{k}\right\|^{1+\beta}\right)$. By using, for $t$ on $K_{k, j}$, the relation

$$
q_{\Delta_{k}}^{\prime}(t)-f^{\prime}(t)=q_{\Delta_{k}}^{\prime}\left(t_{k, j}\right)-f^{\prime}\left(t_{k, j}\right)+\int_{t_{k, j}}^{t}\left[q_{\Delta_{k}}^{\prime \prime}(\tau)-f^{\prime \prime}(\tau)\right] d \tau
$$

and by a repetition of this argument for $q_{\Delta_{k}}(t)-f(t)$ we obtain 
COROLlaRY 1. Under the conditions of Theorem 2, if $f^{\prime \prime}(t)$ satisfies a Hölder condition of order $\beta$ on $K(0<\beta \leqq 1)$, then $\left[q_{\Delta_{k}}^{(p)}(t)-f^{(p)}(t)\right]=O\left(\left\|\Delta_{k}\right\|^{2+\beta-p}\right)$ $(p=0,1,2)$. If $f^{\prime \prime}(t)$ is continuous on $K$, then $\left[q_{\Delta_{k}}^{(p)}(t)-f^{(p)}(t)\right]=o\left(\left\|\Delta_{k}\right\|^{2-p}\right)$.

We have also

CoRollary 2. If $f^{\prime \prime}(t)$ satisfies a Hölder condition of order $\beta(0<\beta \leqq 1)$, then $\left[q_{\Delta_{k}}^{\prime \prime}(t)-f^{\prime \prime}(t)\right] /\left\|\Delta_{k}\right\|^{\beta-\delta}$ satisfies a Hölder condition of order $\delta$ on $K(0<\delta \leqq \beta)$ provided $\left[\max _{j}\left\|\Delta_{k}\right\| /\left|h_{k, j}\right|\right] \leqq C_{1}<\infty$.

Proof. We have, for $t$ and $\tau$ on $K_{j}$ (dropping mesh index $k$ ),

$$
\left|\left[q_{\Delta}^{\prime \prime}(t)-f^{\prime \prime}(t)\right]-\left[q_{\Delta}^{\prime \prime}(\tau)-f^{\prime \prime}(\tau)\right]\right|=|t-\tau|\left|\frac{M_{j}-M_{j-1}}{h_{j}}-\frac{f^{\prime \prime}(t)-f^{\prime \prime}(\tau)}{t-\tau}\right| .
$$

Since $M_{j}-M_{j-1}=O\left(\|\Delta\|^{\beta}\right)$, it is seen that the left-hand member of (17) does not exceed

$$
|t-\tau|^{\delta}\|\Delta\|^{\beta-\delta}\left\{\frac{|t-\tau|^{1-\delta}\|\Delta\|^{\delta}}{\left|h_{j}\right|} \cdot\left[\frac{M_{j}-M_{j-1}}{\|\Delta\|^{\beta}}+\frac{f^{\prime \prime}(t)-f^{\prime \prime}(\tau)}{|t-\tau|^{\beta}} \cdot \frac{\left|h_{j}\right|}{\|\Delta\|^{\beta}|t-\tau|^{1-\beta}}\right]\right\},
$$

and that the term within braces is uniformly bounded with respect to the meshes $\Delta$.

Without the mesh restriction (11) we have

COROLlaRY 3. If $f(z)$ is of class $C^{1}$ on $K$, then $\left[q_{\Delta_{k}}^{\prime}(t)-f^{\prime}(t)\right] /\left\|\Delta_{k}\right\|^{1-\delta}$ satisfies a Hölder condition of order $\delta$ on $K, 0<\delta \leqq 1$.

Proof. Since by (1) we have for $t$ and $\tau$ on $K_{k, j}$

$$
q_{\Delta_{k}}^{\prime}(t)-q_{\Delta_{k}}^{\prime}(\tau)=(t-\tau)\left\{M_{k, j-1} \frac{2 t_{k, j}-t-\tau}{2 h_{k, j}}+M_{j} \frac{t+\tau-2 t_{k, j-1}}{2 h_{k, j}}\right\}
$$

it follows that, for $\left\|\Delta_{k}\right\|$ sufficiently small,

$$
\begin{aligned}
& \left|\left[q_{\Delta_{k}}^{\prime}(t)-f^{\prime}(t)\right]-\left[q_{\Delta_{k}}^{\prime}(\tau)-f^{\prime}(\tau)\right]\right| \\
& \quad=|t-\tau|^{\delta}\left\|\Delta_{k}\right\|^{1-\delta} \cdot\left\{\left|M_{k, j-1}\right|+\left|M_{k, j}\right|+\left|\frac{f^{\prime}(\tau)-f^{\prime}(t)}{\tau-t}\right|\right\} .
\end{aligned}
$$

Sharma and Meir [28] have announced the results of Theorem 3 for the case of a real nonperiodic spline with special end conditions. The proof given above is readily adapted to a wide class of splines including that of Sharma and Meir.

The third derivatives of the spline have jump discontinuities at the junctions $t_{j}$ of the mesh $\Delta$; nevertheless they possess important convergence properties relative to the function approximated. Some of these have been demonstrated by Birkhoff and deBoor [17] for the special spline later considered by Sharma and Meir. Their proofs are rather involved. We study the problem here for the complex cubic splines (periodic) $q_{\Delta}(t)$, giving a simple proof which is readily adapted to real splines of general type.

A somewhat more surprising result is the relation of the jumps in $q_{\Delta}^{m}(t)$ to $f^{\mathrm{iv}}(t)$ when the latter is continuous. This is presented in Theorem 5. 
THEOREM 4. Let $f^{m}(t)$ be continuous on $K$. Let $\left\{\Delta_{k}\right\}$ be a sequence of meshes on $K$ with $\lim _{k \rightarrow \infty}\left\|\Delta_{k}\right\|=0$. Let $q_{\Delta_{k}}(t)$ be the complex cubic spline of interpolation to $f(t)$ on $\Delta_{k}$. If $\left[\max _{j}\left\|\Delta_{k}\right\| /\left|h_{k, j}\right|\right]$ is uniformly bounded with respect to $k$, then $\left\{q_{\Delta_{k}}^{m}(t)\right\} \rightarrow f^{m}(t)$ uniformly on $K$ as $k \rightarrow \infty$.

Proof. Set $\sigma_{j}=\left(M_{j}-M_{j-1}\right) / h_{j}$. In equations (3) we subtract the $(j-1)$ th equation from the $j$ th. Noting that

$$
\begin{gathered}
\frac{h_{j-1}}{h_{j-1}+h_{j}+h_{j+1}}=\frac{\mu_{j-1} \mu_{j}}{1-\mu_{j-1} \lambda_{j}}, \quad \frac{h_{j}}{h_{j-1}+h_{j}+h_{j+1}}=\frac{\lambda_{j-1} \mu_{j}}{1-\mu_{j-1} \lambda_{j}}, \\
\frac{h_{j+1}}{h_{j-1}+h_{j}+h_{j+1}}=\frac{\lambda_{j-1} \lambda_{j}}{1-\mu_{j-1} \lambda_{j}},
\end{gathered}
$$

we set

$$
C=\left[\begin{array}{ccccc}
\frac{\left(1+\mu_{N}+\lambda_{1}\right) \lambda_{N} \mu_{1}}{1-\mu_{N} \lambda_{1}} & \frac{\lambda_{N} \lambda_{1}^{2}}{1-\mu_{N} \lambda_{1}} & 0 & \ldots & \frac{\mu_{N}^{2} \mu_{1}}{1-\mu_{N} \lambda_{1}} \\
\frac{\mu_{1}^{2} \mu_{2}}{1-\mu_{1} \lambda_{2}} & \frac{\left(1+\mu_{1}+\lambda_{2}\right) \lambda_{1} \mu_{2}}{1-\mu_{1} \lambda_{2}} & \frac{\lambda_{1} \lambda_{2}^{2}}{1-\mu_{1} \lambda_{2}} & \ldots & 0 \\
\vdots & & & & \\
\frac{\lambda_{N-1} \lambda_{N}^{2}}{1-\mu_{N-1} \mu_{N}} & 0 & \ldots & \frac{\mu_{N-1}^{2} \mu_{N}}{1-\mu_{N-1} \lambda_{N}} & \frac{\left(1+\mu_{N-1}+\lambda_{N}\right) \lambda_{N-1} \mu_{N}}{1-\mu_{N-1} \lambda_{N}}
\end{array}\right] .
$$

Then

$$
C \sigma=6 \mathrm{r},
$$

where $\sigma=\left(\sigma_{1}, \sigma_{2}, \ldots, \sigma_{N}\right)^{\top}$ and $\mathbf{r}=\left(r_{1}, r_{2}, \ldots, r_{N}\right)^{\top}$, with $r_{j}=f\left[t_{j-2}, t_{j-1}, t_{j}, t_{j+1}\right]$. We may write

$$
G C H=E,
$$

where $G$ and $H$ are the diagonal matrices

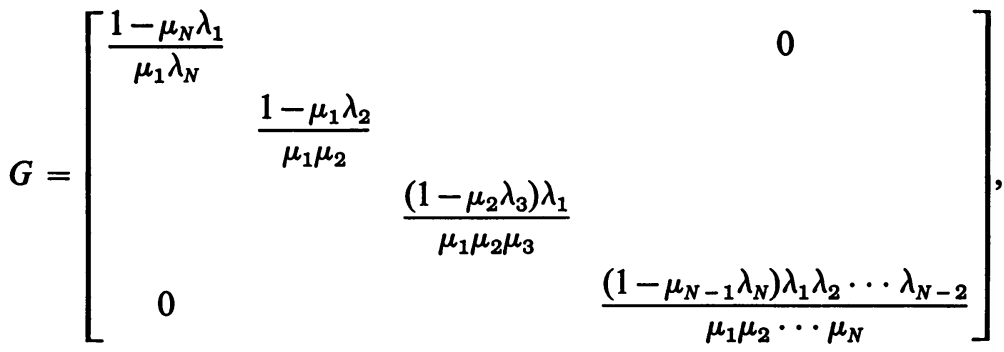

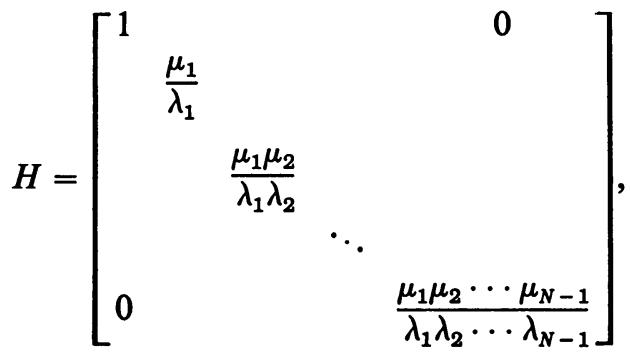


and, since $\lambda_{1} \lambda_{2} \cdots \lambda_{N}=\mu_{1} \mu_{2} \cdots \mu_{N}$,

$$
E=\left[\begin{array}{ccccc}
1+\mu_{N}+\lambda_{1} & \lambda_{1} & 0 & \ldots & \mu_{N} \\
\mu_{1} & 1+\mu_{1}+\lambda_{2} & \lambda_{2} & \ldots & 0 \\
\vdots & & & & \\
0 & \ldots & \mu_{N-2} & 1+\mu_{N-2}+\lambda_{N-1} & \lambda_{N-1} \\
\lambda_{N} & \ldots & 0 & \mu_{N-1} & 1+\mu_{N-1}+\lambda_{N}
\end{array}\right] .
$$

Now $\left\|E^{-1}\right\| \leqq 1+\eta$ (arbitrary $\eta>0$ ) provided $\|\Delta\|$ is sufficiently small. We assume, for all meshes $\Delta$, that we have $\|\Delta\| / \min _{j}\left|h_{j}\right| \leqq C_{1}<\infty$. We find, for arbitrary positive integers $i$ and $p$, that

$$
C_{1}^{-1} \leqq \frac{\lambda_{i}}{\mu_{i}} \cdot \frac{\lambda_{i+1}}{\mu_{i+1}} \cdots \frac{\lambda_{i+p}}{\mu_{i+p}}=\left|\frac{h_{i+p+1}}{h_{i}}\right| \leqq C_{1},
$$

and that for $\|\Delta\|$ sufficiently small,

$$
\left|\mu_{i}\right|=\frac{\left|h_{i}\right|}{\left|h_{i}+h_{i+1}\right|} \leqq \frac{C_{1}+\eta}{1+C_{1}}, \quad\left|\lambda_{i}\right| \leqq \frac{C_{1}+\eta}{1+C_{1}},
$$

from the smoothness of the curve $K$. We require $\eta<1$. Then

$$
\frac{1}{\left|1-\mu_{i-1} \lambda_{i}\right|} \leqq \frac{1}{1-\left(C_{1}+\eta\right)^{2} /\left(1+C_{1}\right)^{2}}=\frac{\left(1+C_{1}\right)^{2}}{(1-\eta)\left(2 C_{1}+\eta+1\right)} .
$$

It follows that $\left\|C^{-1}\right\| \leqq\left(1+C_{1}\right)^{2}(1+\eta) C_{1}^{2} /\left[(1-\eta)\left(2 C_{1}+\eta+1\right)\right]$.

Write (19) as

$$
C(\sigma-6 \mathbf{r})=6(I-C) \mathbf{r} .
$$

The sum of the elements of each row of $I-C$ is zero. Thus the $j$ th component of $(I-C) \mathbf{r}$ is

$$
-\frac{\lambda_{j-1} \lambda_{j}^{2}}{1-\mu_{j-1} \lambda_{j}}\left(r_{j+1}-r_{j}\right)+\frac{\mu_{j-1}^{2} \mu_{j}}{1-\mu_{j-1} \lambda_{j}}\left(r_{j}-r_{j-1}\right) .
$$

Taylor's Theorem with integral remainder gives

$$
f(t)=f\left(t_{j}\right)+\left(t-t_{j}\right) f^{\prime}\left(t_{j}\right)+\left(t-t_{j}\right)^{2} f^{\prime \prime}\left(t_{j}\right) / 2 !+\frac{1}{2 !} \int_{t_{j}}^{t}(t-\tau)^{2} f^{\prime \prime}(\tau) d \tau .
$$

Thus we obtain

$$
\begin{aligned}
& f\left[t_{j-1}, t_{j}, t_{j+1}\right] \\
& =\frac{f_{j}^{\prime \prime}}{2}+\frac{\left(1 / h_{j+1}\right) \int_{t_{j}}^{t_{j+1}}\left(t_{j+1}-\tau\right)^{2} f^{m}(\tau) d \tau-\left(1 / h_{j}\right) \int_{t_{j-1}}^{t_{j}}\left(t_{j-1}-\tau\right)^{2} f^{m}(\tau) d \tau}{2\left(h_{j}+h_{j+1}\right)}
\end{aligned}
$$


and

$$
\begin{aligned}
& f\left[t_{j-2}, t_{j-1}, t_{j}, t_{j+1}\right]-\frac{\left(2 h_{j-1}+h_{j}\right) f_{j-1}^{m}+\left(h_{j}+2 h_{j+1}\right) f_{j}^{m}}{12\left(h_{j-1}+h_{j}+h_{j+1}\right)} \\
&=\frac{1}{2\left(h_{j-1}+h_{j}+h_{j+1}\right)}\left\{\int_{t_{j-1}}^{t_{j}}\left[f^{m}(\tau)-\frac{f_{j-1}^{\prime \prime}+f_{j}^{\prime \prime}}{2}\right] d \tau\right. \\
&+\frac{1}{h_{j}+h_{j+1}}\left[\frac{1}{h_{j+1}} \int_{t_{j}}^{t_{j+1}}\left(t_{j+1}-\tau\right)^{2}\left[f^{m}(\tau)-f_{j}^{m}\right] d \tau\right. \\
&+\frac{1}{h_{j-1}+h_{j}}\left[-\frac{1}{h} \int_{t_{j-1}}^{t_{j}}\left(t_{j}-\tau\right)^{2}\left[f^{m}(\tau)-f_{j-1}^{m}\right] d \tau\right. \\
&\left.\left.+\frac{1}{h_{j-1}} \int_{t_{j-2}}^{t_{j-1}}\left(t_{j-2}-\tau\right)^{2}\left[f^{m}(\tau)-f_{j-1}^{m}\right] d \tau\right]\right\} .
\end{aligned}
$$

Now if we write $t_{j+1}-\tau=\rho e^{i \phi}$, then $|d \tau|=|1+\rho i d \phi / d \rho||d \rho|$ and $|d \phi| d \rho \mid \leqq H_{1}$ on $K$ for some constant $H_{1}$. Thus

$$
\left(1 /\left|h_{j+1}\right|\right) \int_{t_{j}}^{t_{j+1}}\left|t_{j+1}-\tau\right|^{2}|d \tau| \leqq\left|h_{j+1}\right|^{2}\left(1 / 3+H_{1}\left|h_{j+1}\right| / 4\right) .
$$

It follows that the left-hand member of (22) is in absolute value not greater than

$$
\frac{\mu\left(\|\Delta\|, f^{\prime \prime}\right)}{2\left|h_{j-1}+h_{j}+h_{j+1}\right|}\left\{1+\frac{\left|h_{j}\right|^{2}+\left|h_{j+1}\right|^{2}}{\left|h_{j}+h_{j+1}\right|}+\frac{\left|h_{j-1}\right|^{2}+\left|h_{j}\right|^{2}}{\left|h_{j-1}+h_{j}\right|}\right\} \cdot\left(\frac{1}{3}+\frac{H_{1}\|\Delta\|}{4}\right) .
$$

Here the coefficient of $\mu\left(\|\Delta\|, f^{m}\right)$ is seen to be dominated by a function of $H_{1}$ alone provided $\|\Delta\|$ is sufficiently small.

The left-hand member of (22) is the difference between $r_{j}$ and a weighted mean of $f_{j-1}^{m}$ and $f_{j}^{m}$. We may conclude that $\|(I-C) \mathbf{r}\| \rightarrow 0$ as $\|\Delta\| \rightarrow 0$. From the uniform boundedness of $\left\|C^{-1}\right\|$ we have $\|\sigma-6 \mathbf{r}\| \rightarrow 0$ by $(21)$ and $\left\{q^{m}(t)\right\} \rightarrow f^{m}(t)$ uniformly on $K$ as $\|\Delta\| \rightarrow 0\left(q^{m}(t)\right.$ is constant on each $K_{j}$ and $f^{m}(t)$ is uniformly continuous on $K)$. This completes the proof of the theorem.

If $f^{\prime \prime}(t)$ satisfies a Hölder condition of order $\beta$ on $K(0<\beta \leqq 1)$, then the quantities (21) may be shown to be less than $b \cdot\|\Delta\|^{\beta}$ for some constant $b$ independent of $\Delta$. Thus $f^{m}(t)-q_{\Delta}^{m}(t)=O\left(\|\Delta\|^{\beta}\right)$.

To obtain the degree of convergence of $\left\{q_{\Delta_{k}}^{\prime \prime}(t)\right\}$ we must circumvent the difficulty that $q_{\Delta_{k}}^{m}(t)$ is not continuous. We have

$$
\begin{aligned}
& \frac{\left(f_{j+1}-f_{j}\right) / h_{j+1}-\left(f_{j}-f_{j-1}\right) / h_{j}}{h_{j}+h_{j+1}} \\
& \quad=\frac{f_{j}^{\prime \prime}}{2}+\frac{\left(1 / h_{j+1}\right) \int_{t_{j}}^{t_{j+1}}\left(t_{j+1}-\tau\right)^{2} f^{m}(\tau) d \tau-\left(1 / h_{j}\right) \int_{t_{j-1}}^{t_{j}}\left(t_{j-1}-\tau\right)^{2} f^{m}(\tau) d \tau}{2\left(h_{j}+h_{j+1}\right)} .
\end{aligned}
$$


Using (3) and the interpolation property of $q_{\Delta}(t)$, we obtain

$$
\begin{aligned}
f_{j}^{n}- & M_{j} \\
& =\frac{\left(1 / h_{j+1}\right) \int_{t_{j}+1}^{t_{j+1}}\left(t_{j+1}-\tau\right)^{2}\left[f^{m}(\tau)-\sigma_{j+1}\right] d \tau-\left(1 / h_{j}\right) \int_{t_{j-1}}^{t_{j}}\left(t_{j-1}-\tau\right)^{2}\left[f^{m}(\tau)-\sigma_{j}\right] d \tau}{h_{j}+h_{j+1}} .
\end{aligned}
$$

Thus $f_{j}^{\prime \prime}-M_{j}=O\left(\|\Delta\|^{1+\beta}\right)$ and, for $t$ on $K_{j}$,

$$
f^{\prime \prime}(t)-q_{\Delta}^{\prime \prime}(t)=f_{j}^{\prime \prime}-M_{j}+\int_{t_{j}}^{t}\left[f^{m}(\tau)-\sigma_{j}\right] d \tau,
$$

so that $\left[f^{\prime \prime}(t)-q_{\Delta}^{\prime \prime}(t)\right]=O\left(\|\Delta\|^{1+\beta}\right)$.

The corresponding convergence rates for $\left[f^{\prime}(t)-q_{\Delta}^{\prime}(r)\right]$ and $\left[f(t)-q_{\Delta}(t)\right]$ now result by integration as before and we have

COROLlaRY 1. Under the conditions of Theorem 4, let $f^{m}(t)$ satisfy a Hölder condition of order $\beta(0<\beta \leqq 1)$ on $K$. Then $q_{\Delta_{k}}^{(p)}(t)-f^{(p)}(t)=O\left(\left\|\Delta_{k}\right\|^{3+\beta-p}\right), p=0,1$, 2, 3. If $f^{m}(t)$ is continuous on $K$, then $\left[q_{\Delta_{k}}^{(p)}(t)-f^{(p)}(t)\right]=o\left(\left\|\Delta_{k}\right\|^{3-p}\right)$.

The function $q^{m}(t)-f^{m}(t)$ does not, of course, satisfy a Hölder condition on $K$. We do, however, have

COROLlARY 2. If $f^{m}(z)$ satisfies a Hölder condition of order $\beta(0<\beta \leqq 1)$, then for arbitrary $\delta(0<\delta \leqq \beta)$ the functions $\left[q_{\Delta_{k}}^{\prime \prime}(t)-f^{\prime \prime}(t)\right] /\left\|\Delta_{k}\right\|^{1+\beta-\delta}$ satisfy a Hölder condition of order $\delta$ uniformly with respect to $k$.

Proof. We have on $K_{k, j}$

$$
\left|\left[q_{\Delta_{k}}^{\prime \prime}(t)-f^{\prime \prime}(t)\right]-\left[q_{\Delta_{k}}^{\prime \prime}(\tau)-f^{\prime \prime}(\tau)\right]\right|=|t-\tau|\left|\sigma_{k, 5}-\frac{f^{\prime \prime}(t)-f^{\prime \prime}(\tau)}{t-\tau}\right| .
$$

Now

$$
\left|f^{m}(t)-\frac{f^{\prime \prime}(t)-f^{\prime \prime}(\tau)}{t-\tau}\right|=\frac{1}{|t-\tau|}\left|\int_{\tau}^{t}\left[f^{m}(t)-f^{m}(\tau)\right] d \tau\right|
$$

is $O\left(\left\|\Delta_{k}\right\|^{\beta}\right)$ and so is $\left|\sigma_{k, j}-f^{m}(t)\right|$ by Corollary 1 . Thus the left-hand member of (23) does not exceed

$$
|t-\tau| \cdot\left\|\Delta_{k}\right\|^{1+\beta-\delta} \cdot \frac{|t-\tau|^{1-\delta}}{\left\|\Delta_{k}\right\|^{1-\delta}} \cdot\left|\frac{\sigma_{k, j}-f^{m}(t)}{\left\|\Delta_{k}\right\|^{\beta}}+\frac{f^{m}(t)-\left[f^{\prime \prime}(t)-f^{\prime \prime}(\tau)\right] /(t-\tau)}{\left\|\Delta_{k}\right\|^{\beta}}\right| .
$$

The corollary follows.

COROLlaRY 3. Under the conditions of Theorem 4 but without the additional mesh restriction (11), for arbitrary $\delta(0<\delta \leqq 1)$ the functions

$$
\left[q_{\Delta_{k}}^{\prime \prime}(t)-f^{\prime \prime}(t)\right] /\left\|\Delta_{k}\right\|^{1-\delta}
$$

satisfy a Hölder condition of order $\delta$ on $K$ uniformly with respect to $k$. 
Proof. The right-hand member of (23) does not exceed

$$
|t-\tau|^{\delta}\left\|\Delta_{k}\right\|^{1-\delta}\left[\sup _{k} \max _{j}\left|\sigma_{k, j}\right|+\sup _{K}\left|\frac{f^{\prime \prime}(t)-f^{\prime \prime}(\tau)}{t-\tau}\right|\right]
$$

and the bracketed expression is finite. This completes the proof.

Consider next the jump in $q_{\Delta}^{m}(t)$ at the junctions $t_{j}$ of $\Delta$. Define

$$
\delta_{j}=\frac{\sigma_{j+1}-\sigma_{j}}{h_{j}+h_{j+1}}
$$

Subtract each of the component equations of (19) from its successor and obtain $(j=1,2, \ldots, N)$ the equation

$$
\begin{aligned}
\frac{\mu_{j-1}^{2} \mu_{j}}{1-\mu_{j-1} \lambda_{j}}\left(\sigma_{j}-\sigma_{j-1}\right)+\mu_{j} \lambda_{j}\left(\frac{1}{1-\mu_{j-1} \lambda_{j}}\right. & \left.+\frac{1}{1-\mu_{j} \lambda_{j+1}}\right)\left(\sigma_{j+1}-\sigma_{j}\right) \\
& +\frac{\lambda_{j} \lambda_{j+1}^{2}}{1-\mu_{j} \lambda_{j+1}}\left(\sigma_{j+2}-\sigma_{j+1}\right)=6\left(r_{j+1}-r_{j}\right) .
\end{aligned}
$$

Note that

$$
\frac{\lambda_{j-1} \lambda_{j}+\mu_{j} \mu_{j+1}}{h_{j-1}+h_{j}+h_{j+1}+h_{j+2}}=\frac{\mu_{j} \mu_{j+1}}{h_{j-1}+h_{j}}=\frac{\lambda_{j-1} \mu_{j+1}}{h_{j}+h_{j+1}}=\frac{\lambda_{j} \lambda_{j-1}}{h_{j+1}+h_{j+2}} .
$$

Set $\theta_{j}=\left(1-\mu_{j-1} \lambda_{j}\right)^{-1}$ and

$$
D=\left[\begin{array}{ccccc}
\frac{\lambda_{N} \lambda_{1} \mu_{1} \mu_{2}\left(\theta_{1}+\theta_{2}\right)}{\lambda_{N} \lambda_{1}+\mu_{1} \mu_{2}} & \frac{\lambda_{N} \lambda_{1}^{2} \lambda_{2}^{2} \theta_{2}}{\lambda_{N} \lambda_{1}+\mu_{1} \mu_{2}} & 0 & \ldots & \frac{\mu_{N}^{2} \mu_{1}^{2} \mu_{2} \theta_{1}}{\lambda_{N} \lambda_{1}+\mu_{1} \mu_{2}} \\
\frac{\mu_{1}^{2} \mu_{2}^{2} \mu_{3} \theta_{2}}{\lambda_{1} \lambda_{2}+\mu_{2} \mu_{3}} & \frac{\lambda_{1} \lambda_{2} \mu_{2} \mu_{3}\left(\theta_{2}+\theta_{3}\right)}{\lambda_{1} \lambda_{2}+\mu_{2} \mu_{3}} & \frac{\lambda_{1} \lambda_{2}^{2} \lambda_{3}^{2} \theta_{3}}{\lambda_{1} \lambda_{2}+\mu_{2} \mu_{3}} & \ldots & 0 \\
\vdots & & & & 0 \\
\frac{\lambda_{N-1} \lambda_{N}^{2} \lambda_{1}^{2} \theta_{1}}{\lambda_{N-1} \lambda_{N}+\mu_{N} \mu_{1}} & 0 & \ldots & \frac{\mu_{N-1}^{2} \mu_{N}^{2} \mu_{1} \theta_{N}}{\lambda_{N-1} \lambda_{N}+\mu_{N} \mu_{1}} & \frac{\lambda_{N-1} \lambda_{N} \mu_{N} \mu_{1}\left(\theta_{N}+\theta_{1}\right)}{\lambda_{N-1} \lambda_{N}+\mu_{N} \mu_{1}}
\end{array}\right] ;
$$

also let $\delta=\left(\delta_{1}, \delta_{2}, \ldots, \delta_{N}\right)^{\top}$ and $\mathbf{d}=\left(d_{1}, d_{2}, \ldots, d_{N}\right)^{\top}$, where

Then

$$
d_{j}=6 f\left[t_{j-2}, t_{j-1}, t_{j}, t_{j+1}, t_{j+2}\right] \text {. }
$$

$$
D \boldsymbol{\delta}=\mathbf{d} .
$$

As in the proof of Theorem 4 we transform $D$ into a matrix with dominant main diagonal by left and right multiplication by suitable diagonal matrices. We multiply the rows of $D$ by

$$
\begin{aligned}
& \left(\lambda_{N} \lambda_{1}+\mu_{1} \mu_{2}\right) \lambda_{N} /\left(\mu_{1}^{2} \mu_{2}\right),\left(\lambda_{1} \lambda_{2}+\mu_{2} \mu_{3}\right) \lambda_{N}^{2} \lambda_{1} /\left(\left(\mu_{1} \mu_{2}\right)^{2} \mu_{3}\right), \ldots, \\
& \left(\lambda_{N-2} \lambda_{N-1}+\mu_{N-1} \mu_{N}\right)\left(\lambda_{N} \lambda_{1} \cdots \lambda_{N-3}\right)^{2} \lambda_{N-2} /\left(\left(\mu_{1} \cdots \mu_{N-1}\right)^{2} \mu_{N}\right) \\
& \quad=\left(\lambda_{N-2} \lambda_{N-1}+\mu_{N-1} \mu_{N}\right) \mu_{N} /\left(\lambda_{N-1}^{2} \lambda_{N-2}\right),\left(\lambda_{N-1} \lambda_{N}+\mu_{N} \mu_{1}\right) /\left(\lambda_{N-1} \mu_{1}\right),
\end{aligned}
$$

and the columns by

$$
\begin{gathered}
\mu_{1} /\left(\lambda_{N}^{2} \lambda_{1}\right), \mu_{1}^{2} \mu_{2} /\left(\left(\lambda_{N} \lambda_{1}\right)^{2} \lambda_{2}\right), \ldots,\left(\mu_{1} \cdots \mu_{N-2}\right)^{2} \mu_{N-1} /\left(\left(\lambda_{N} \lambda_{1} \cdots \lambda_{N-2}\right)^{2} \lambda_{N-1}\right) \\
=\lambda_{N-1} /\left(\mu_{N-1} \mu_{N}^{2}\right), 1 /\left(\lambda_{N} \mu_{N}\right)
\end{gathered}
$$


We obtain as a result the matrix

$$
\left[\begin{array}{ccccccc}
\theta_{1}+\theta_{2} & \lambda_{2} \theta_{2} & 0 & \ldots & 0 & 0 & \mu_{N} \theta_{1} \\
\mu_{1} \theta_{2} & \theta_{2}+\theta_{3} & \lambda_{3} \theta_{3} & \ldots & 0 & 0 & 0 \\
\vdots & & & & & & \\
0 & 0 & \ldots & \ldots & \mu_{N-2} \theta_{N-1} & \theta_{N-1}+\theta_{N} & \lambda_{N} \theta_{N} \\
\lambda_{1} \theta_{1} & 0 & \ldots & \ldots & 0 & \mu_{N-1} \theta_{N} & \theta_{N}+\theta_{1}
\end{array}\right] .
$$

If we assume for the meshes $\Delta$ under consideration that $\|\Delta\| / \min _{j}\left|h_{j}\right| \leqq C^{*}<\infty$, we find by the methods used previously that $\left\|D^{-1}\right\|$ is uniformly bounded (the matrix (25) has dominant main diagonal for $\|\Delta\|$ sufficiently small).

Now consider the sum of elements in the $j$ th row of $D$,

$$
\begin{aligned}
G_{j} & =\frac{\mu_{j-1}^{2} \mu_{j}^{2} \mu_{j+1} \theta_{j}+\lambda_{j-1} \lambda_{j} \mu_{j} \mu_{j+1}\left(\theta_{j}+\theta_{j+1}\right)+\lambda_{j-1} \lambda_{j}^{2} \lambda_{j+1}^{2} \theta_{j+1}}{\lambda_{j-1} \lambda_{j}+\mu_{j} \mu_{j+1}} \\
& =\frac{\left(\mu_{j-1}^{2} \mu_{j} / \lambda_{j-1} \lambda_{j}\right) \theta_{j}+\left(\theta_{j}+\theta_{j+1}\right)+\left(\lambda_{j} \lambda_{j+1}^{2} / \mu_{j} \mu_{j+1}\right) \theta_{j+1}}{1 / \mu_{j} \mu_{j+1}+1 / \lambda_{j-1} \lambda_{j}} .
\end{aligned}
$$

If the meshes $\Delta$ become asymptotically uniform as $k \rightarrow \infty$; that is, if

$$
\max _{j}\left|\lambda_{j}-1 / 2\right| \rightarrow 0
$$

then $\max _{j}\left|G_{j}-1 / 2\right| \rightarrow 0$. In general, let $G$ be the diagonal matrix with $G_{j}$ the diagonal element in the $j$ th row. Then for $G^{-1} D-I$ the sum of elements in each row is zero $\left(G_{j} \neq 0\right.$ for $\|\Delta\|$ sufficiently small).

We write (24) as

$$
D \delta-G^{-1} D \mathbf{d}=\left(I-G^{-1} D\right) \mathbf{d} .
$$

Let $f^{\mathrm{iv}}(t)$ be continuous on $K$. The quantities $4 d_{j}$ differ from a weighted mean of $f_{j-1}^{\mathrm{iv}}, f_{j}^{\mathrm{iv}}, f_{j-1}^{\mathrm{iv}}$ by an amount which is $O(\|\Delta\|)$. This follows from the uniform continuity of $f^{\mathrm{iv}}$ and properties of the $d_{j}$. Thus $\left\|\left(I-G^{-1} D\right) \mathbf{d}\right\| \rightarrow 0$ as $\|\Delta\| \rightarrow 0$. Since $\left\|D^{-1}\right\|$ is uniformly bounded, it is evident that $\left\|\delta-D^{-1} G^{-1} D \mathbf{d}\right\| \rightarrow 0$. If the meshes become asymptotically uniform as $\|\Delta\| \rightarrow 0$, then $\|\boldsymbol{\delta}-2 \mathbf{d}\| \rightarrow 0$. Thus we have

THEOREM 5. If $f^{\mathrm{iv}}(t)$ is continuous on $K$ and if, as $\left\|\Delta_{k}\right\| \rightarrow 0$, we have

$$
\left[\left\|\Delta_{k}\right\| / \min _{j}\left|h_{k, j}\right|\right] \leqq C<\infty,
$$

then $\left\|\delta_{k}-D_{k}^{-1} G_{k}^{-1} D_{k} \mathbf{d}_{k}\right\| \rightarrow 0$. If the meshes become asymptotically uniform as $\left\|\Delta_{k}\right\| \rightarrow 0$, then

$$
\lim _{\left\|\Delta_{k}\right\| \rightarrow 0}\left[\max _{j}\left|\frac{q_{\Delta_{k}}^{m}\left(t_{k, j}+\right)-q_{\Delta_{k}}^{m}\left(t_{k, j}-\right)}{h_{j}+h_{j+1}}-\frac{f^{\mathrm{1v}}\left(t_{k, j}\right)}{2}\right|\right]=0 .
$$

Finally we show that the convergence rate of $q_{\Delta_{k}}^{(p)}(t)-f^{(p)}(t)$ to zero can be no higher than $O\left(\left\|\Delta_{k}\right\|^{4-p}\right)$. Let us assume $f^{\mathrm{iv}}(t)$ to be continuous on $K$ and that 
$\left|q_{\Delta_{k}}^{(p)}(t)-f^{(p)}(t)\right| \leqq B_{1}\left\|\Delta_{k}\right\|^{4-p+\mu}$ for some $\mu>0$ and some $p(p=0,1,2,3$, or 4$)$. If $p=4$, then $f^{\mathrm{iv}}(t) \equiv 0$. If $p=3$, then as in the proof of Corollary I of Theorem 4 we have $q_{\Delta_{k}}^{\prime \prime}(t)-f^{\prime \prime}(t)=O\left(\left\|\Delta_{k}\right\|^{2+\mu}\right)$; hence, as in the proof of Corollary 1 of Theorem 3, $q_{\Delta_{k}}^{(p)}(t)-f^{(p)}(t)=O\left(\left\|\Delta_{k}\right\|^{4+\mu-p}\right)$ for $p=0,1$. In all cases, therefore, we are led to the situation in which $\left|q_{\Delta k}(t)-f(t)\right| \leqq B\left\|\Delta_{k}\right\|^{4+\mu}$. Subdivide each interval $\left[t_{k, j-1}, t_{k, j}\right]$ into four subintervals of equal length by points $\xi_{j, 0}=t_{j-1}, \xi_{j, 1}, \xi_{j, 2}, \xi_{j, 3}, \xi_{j, 4}=t_{j}$ (dropping mesh index $k$ ); set $h_{j, n}=\xi_{j, n}-\xi_{j, n-1}(n=1,2,3,4)$. Form the difference of fourth divided differences

$$
q_{\Delta}\left[\xi_{j, 0}, \xi_{j, 1}, \ldots, \xi_{j, 4}\right]-f\left[\xi_{j, 0}, \xi_{j, 1}, \ldots, \xi_{j, 4}\right]=\left(q_{\Delta}-f\right)\left[\xi_{j, 0}, \xi_{j, 1}, \ldots, \xi_{j, 4}\right] .
$$

The right-hand member of this equation does not exceed

$$
\left(\|\Delta\| /\left|h_{j}\right|\right)^{4+\mu} \cdot\left(\left|h_{j}\right| / \min _{n}\left|h_{j, n}\right|\right)^{4} \cdot\left|h_{j}\right|^{\mu} / 6 .
$$

If $\|\Delta\| /\left|h_{j}\right| \leqq C_{1}<\infty$, then the right-hand member is $O\left(\|\Delta\|^{\mu}\right)$. Since $q_{\Delta}(t)$ is cubic in $t$ over the interval $t_{j-1}, t_{j}$, and since $f\left[\xi_{j, 0}, \xi_{j, 1}, \ldots, \xi_{j, 4}\right]-f^{i \mathrm{v}}\left(t_{j-1}\right) \rightarrow 0$, we have $f^{1 \mathrm{v}}(t) \equiv 0$ on $K$. Hence

THEOREM 6. Let $f^{\mathrm{iv}}(t)$ be continuous on $K$. Let $\left\{\Delta_{k}\right\}$ be a sequence of meshes on $K$ with $\lim _{k} \rightarrow\left\|\Delta_{k}\right\|=0$ and $\left[\max _{j}\left\|\Delta_{k}\right\| /\left|h_{k, j}\right|\right] \leqq C_{1}<\infty$. If

$$
\left|q_{k}^{(p)}(t)-f^{(p)}(t)\right|=O\left(\left\|\Delta_{k}\right\|^{4+\mu-p}\right)
$$

for any $p(p=0,1,2,3,4)$ and $\mu>0$, then $f^{\mathrm{iv}}(t) \equiv 0$ on $K$.

The analytic spline. Let $f(t)$ be continuous on $K$. Let $\Delta$ be a mesh on $K$ and $q_{\Delta}(t)$ the complex cubic spline of interpolation to $f(t)$ on $\Delta$. If $R$ represents the region interior to $K$, then the complex spline $S_{\Delta}(z), z$ in $R$, is defined by (8).

We note that if for $z$ in $R$ we let $z \rightarrow t$ on $K$, then [29] $S_{\Delta}(z) \rightarrow S_{\Delta}(t)$, where

$$
S_{\Delta}(t)=\frac{1}{2} q_{\Delta}(t)+\frac{1}{2 \pi i} \int_{K} \frac{q_{\Delta}(\tau)}{\tau-t} d \tau .
$$

Here the Cauchy principal value of the integral on the right is intended. On $K$, if $q_{\Delta}(t)-f(t)$ satisfies a Hölder condition of order $\delta>0$, we have

$$
S_{\Delta}(t)-f(t)=\frac{1}{2}\left[q_{\Delta}(t)-f(t)\right]+\frac{1}{2 \pi i} \int_{K} \frac{q_{\Delta}(\tau)-f(\tau)}{\tau-t} d \tau .
$$

From the Corollary to Theorem 1 and Corollary 3 of Theorem 2, together with an application of the Principle of Maximum for analytic functions, we obtain

THEOREM 7. Let $f(z)$ be analytic in $R$ and continuous in $\bar{R}=R+K$. Let $\left\{\Delta_{k}\right\}$ be a sequence of meshes on $K$ with $\lim _{k \rightarrow \infty}\left\|\Delta_{k}\right\|=0$ and $\left[\max _{j}\left\|\Delta_{k}\right\| /\left|h_{k, j}\right|\right] \leqq C_{1}<\infty$. If $f(t)$ satisfies a Hölder condition on $K$ of order $\beta(0<\beta \leqq 1)$, then for $z$ in $\bar{R}$ and any $\beta^{\prime}, 0<\beta^{\prime}<\beta$, we have

$$
S_{\Delta_{k}}(z)-f(z)=O\left(\left\|\Delta_{k}\right\|^{\beta^{\prime}}\right)
$$


If $f^{\prime}(t)$ exists and is continuous on $K$, then (33) is valid for arbitrary $\beta^{\prime}, 0<\beta^{\prime}<1$, with only the restriction $\lim _{k \rightarrow \infty}\left\|\Delta_{k}\right\|=0$ on the meshes $\Delta_{k}$.

Let $f^{\prime}(t)$ satisfy a Hölder condition on $K$. Then we have

$$
S_{\Delta}^{\prime}(t)-f^{\prime}(t)=\frac{1}{2}\left[q_{\Delta}^{\prime}(t)-f^{\prime}(t)\right]+\frac{1}{2 \pi i} \int_{K} \frac{q_{\Delta}^{\prime}(\tau)-f^{\prime}(\tau)}{\tau-t} d \tau
$$

We obtain from Corollary 2 of Theorem 2 and Corollary 3 of Theorem 3 the following:

THEOREM 8. Let $f(z)$ be analytic in $R$ and of Class $C^{1}$ in $\bar{R}$. Let $\left\{\Delta_{k}\right\}$ be a sequence of meshes on $K$ with $\lim _{k \rightarrow \infty}\left\|\Delta_{k}\right\|=0$ and $\max _{j}\left\|\Delta_{k}\right\| /\left|h_{k, j}\right| \leqq C<\infty$. If $f^{\prime}(t)$ satisfies a Hölder condition of order $\beta$ on $K(0<\beta \leqq 1)$, then for $z$ in $\bar{R}$ and any $\beta^{\prime}, 0<\beta^{\prime}<\beta$, we have

$$
S_{\Delta_{k}}^{(p)}(z)-f^{(p)}(z)=O\left(\left\|\Delta_{k}\right\|^{1+\beta^{\prime}-p}\right) \quad(p=0,1) .
$$

If $f^{\prime \prime}(t)$ exists and is continuous on $K$, then (34) is valid for arbitrary $\beta^{\prime}, 0<\beta^{\prime}<1$, with only the restriction $\lim _{k \rightarrow \infty}\left\|\Delta_{k}\right\|=0$ on the meshes $\Delta_{k}$. In this case also $\left[S_{\Delta_{k}}^{(p)}(z)-f^{(p)}(z)\right]=O\left(\left\|\Delta_{k}\right\|^{1+\beta^{\prime}}\right)$ for $p=0,1, \ldots$, on any closed subset of $R$.

The last conclusion of the theorem follows from the Cauchy Integral Formula. By Corollary 2 of Theorem 3 and Corollary 3 of Theorem 4 we are led to conclude the following theorem:

TheOrem 9. Let $f(z)$ be analytic in $R$ and of Class $C^{2}$ in $\bar{R}$. Let $\left\{\Delta_{k}\right\}$ be a sequence of meshes on $K$ with $\lim _{k \rightarrow \infty}\left\|\Delta_{k}\right\|=0$ and $\max _{j}\left\|\Delta_{k}\right\| / h_{k, j} \mid \leqq C<\infty$. If $f^{\prime \prime}(t)$ satisfies a Hölder condition of order $\beta$ on $K(0<\beta \leqq 1)$, then for $z$ in $\bar{R}$ and any $\beta^{\prime}, 0<\beta^{\prime}<\beta$, we have $(p=0,1,2)$

$$
S_{\Delta_{k}}^{(p)}(z)-f^{(p)}(z)=O\left(\left\|\Delta_{k}\right\|^{2+\beta^{\prime}-p}\right) .
$$

If $f^{\prime \prime}(t)$ exists and is continuous on $K$, then (35) is valid for arbitrary $\beta^{\prime}, 0<\beta^{\prime}<1$, without the additional mesh restriction. Here also $S_{\Delta_{k}}^{(p)}(z)-f^{(p)}(z)$ is $O\left(\left\|\Delta_{k}\right\|^{2+\beta^{\prime}}\right)$ $(p=0,1,2)$ on any closed subset of $R$.

If $f(z)$ is analytic in $R$ and of Class $C^{3}$ in $\bar{R}$, we wish to examine the convergence of the sequence $\left\{S_{\Delta_{k}}^{m}(z)\right\}$. The method employed in the preceding three theorems does not apply without modification since $q_{k}^{m}(t)$ is discontinuous at the mesh points $t_{k, y}$. However, using Corollary 2 of Theorem 4 we obtain

THEOREM 10. Let $f(z)$ be analytic in $R$ and of Class $C^{3}$ in $\bar{R}$. Let $\left\{\Delta_{k}\right\}$ be a sequence of meshes on $K$ with $\lim _{k \rightarrow \infty}\left\|\Delta_{k}\right\|=0$, and $\left[\max _{j}\left\|\Delta_{k}\right\| /\left|h_{k, j}\right|\right] \leqq C<\infty$. If $f^{m}(t)$ satisfies a Hölder condition of order $\beta$ on $K, 0<\beta \leqq 1$, then for $z$ in $\bar{R}$ and any $\beta^{\prime}, 0<\beta^{\prime}<\beta$ we have $(p=0,1,2)$

$$
S_{\Delta_{k}}^{(p)}(z)-f^{(p)}(z)=O\left(\left\|\Delta_{k}\right\|^{3+\beta^{\prime}-p}\right)
$$

On any closed subset of $R, S_{\Delta_{k}}^{(p)}(z)-f^{(p)}(z)=O\left(\left\|\Delta_{k}\right\|^{3+\beta^{\prime}}\right)(p=0,1,2,3)$. 
Structure of the analytic spline. The integration indicated in (8) may be carried out explicitly. We have

$$
2 \pi i S_{\Delta}(z)=\sum_{j=1}^{N} \int_{K,} \frac{q_{\Delta, j}(\tau)}{\tau-z} d \tau,
$$

when $q_{\Delta, j}(t)$ is the cubic given by (1) or (5) which coincides with $q_{\Delta}(t)$ on the arc $K_{j}$. Employing (1), rearrange $q_{\Delta, j}(\tau)$ as follows:

$$
\begin{aligned}
q_{\Delta, j}(\tau)= & \frac{M_{j-1}}{6 h_{j}}\left[\left(t_{j}-z\right)^{3}-3\left(t_{j}-z\right)^{2}(\tau-z)+3\left(t_{j}-z\right)(\tau-z)^{2}-(\tau-z)^{3}\right] \\
& +\frac{M_{j}}{6 h_{j}}\left[\left(z-t_{j-1}\right)^{3}+3\left(z-t_{j-1}\right)^{2}(\tau-z)+3\left(z-t_{j-1}\right)(\tau-z)^{2}+(\tau-z)^{3}\right] \\
& +\left(f_{j-1} / h_{j}-M_{j-1} h_{j} / 6\right)\left[\left(t_{j}-z\right)-(\tau-z)\right] \\
& +\left(f_{j} / h_{j}-M_{j} h_{j} / 6\right)\left[\left(z-t_{j-1}\right)+(\tau-z)\right] .
\end{aligned}
$$

We obtain, using $\sum_{j=1}^{N} M_{j}\left(h_{j}+h_{j+1}\right)=0$, the relation

$$
2 \pi i S_{\Delta}(z)=\sum_{j=1}^{N} q_{\Delta, j}(z) \log \frac{t_{j}-z}{t_{j-1}-z}-\frac{5}{36} \sum_{j=1}^{N} M_{j}\left(h_{j}+h_{j+1}\right)\left(t_{j-1}+t_{j}+t_{j+1}\right) .
$$

The quantity $z$ is, of course, generally outside the domain of definition of $q_{\Delta, j}(t)$. Here $q_{\Delta, j}(z)$ represents the result of substituting $z$ for $t$ in (1). Thus

$$
\begin{aligned}
& 2 \pi i S_{\Delta}^{\prime}(z)=\sum_{j=1}^{N} q_{\Delta, j}^{\prime}(z) \log \frac{t_{j}-z}{t_{j-1}-z}-\sum_{j=1}^{N} \frac{\left(t_{j}-z\right)^{2}}{6}\left[\frac{M_{j+1}-M_{j}}{h_{j+1}}-\frac{M_{j}-M_{j-1}}{h_{j}}\right], \\
& 2 \pi i S_{\Delta}^{\prime \prime}(z)=\sum_{j=1}^{N} q_{\Delta, j}^{\prime \prime}(z) \log \frac{t_{j}-z}{t_{j-1}-z}+\sum_{j=1}^{N} \frac{5\left(t_{j}-z\right)}{6}\left[\frac{M_{j+1}-M_{j}}{h_{j+1}}-\frac{M_{j}-M_{j-1}}{h_{j}}\right], \\
& 2 \pi i S_{\Delta}^{\mathrm{m}}(z)=\sum_{j=1}^{N} q_{\Delta, j}^{m}(z) \log \frac{t_{j}-z}{t_{j-1}-z}=\sum_{j=1}^{N} \sigma_{j} \log \frac{t_{j}-z}{t_{j-1}-z}, \\
& 2 \pi i S_{\Delta}^{\mathrm{iv}}(z)=\sum_{j=1}^{N} \frac{\sigma_{j+1}-\sigma_{j}}{t_{j}-z} .
\end{aligned}
$$

We have previously established the fact that, for a sequence of meshes $\left\{\Delta_{k}\right\}$ on $K$ with $\left\|\Delta_{k}\right\| \rightarrow 0,\left[\max _{j}\left\|\Delta_{k}\right\| /\left|h_{k, j}\right|\right] \leqq C<\infty$ and $\left[\max _{j}\left|\lambda_{j}-1 / 2\right|\right] \rightarrow 0$ as $k \rightarrow \infty$, and a function $f(t)$ with $f^{\mathrm{iv}}(t)$ continuous on $K$, we have

$$
\lim _{k \rightarrow \infty}\left[\max _{j} \mid \frac{\sigma_{k, j+1}-\sigma_{k, j}}{h_{k, j}+h_{k, j+1}}-\frac{1}{2} f^{\mathrm{iv}}\left(t_{j}\right)\right] \mid=0 .
$$

If in addition $f(t)$ represents the boundary values of a function $f(z)$ analytic in $R$, then we know from the convergence properties of $\left\{S_{\Delta k}(z)\right\}$ that

$$
\lim _{k \rightarrow \infty} \frac{1}{2 \pi i} \sum_{j=1}^{N_{k}} \frac{\sigma_{k, j+1}-\sigma_{k, j}}{\left(h_{k, j}+h_{k, j+1}\right) / 2} \cdot \frac{1}{t_{j}-z} \cdot \frac{h_{k, j}+h_{k, j+1}}{2}=\frac{1}{2 \pi i} \int_{K} \frac{f^{\mathrm{iv}}(t)}{t-z} d t .
$$

Furthermore, for $z$ on any closed subset of $R$ the rate of convergence is $O\left(\left\|\Delta_{k}\right\|^{4}\right)$. 
This relationship, however, sheds some light on the nature of the spline approximation. The sum in the left-hand member of (37) may be interpreted as a "trapezoidal" approximation of the integral appearing on the right, and more generally relates to the classical theorem by Runge concerning the approximation of a function analytic in $R$ by rational functions with poles on $K$ [30].

The last term in (36) can be further simplified. If we multiply equation (2) by $t_{j}$ and sum over $j$, the term in $M_{j}$ is

$$
M_{j}\left(\frac{h_{j}}{6} t_{j-1}+\frac{h_{j}+h_{j+1}}{3} t_{j}+\frac{h_{j+1}}{6} t_{j+1}\right)=\frac{M_{j}}{6}\left(h_{j}+h_{j+1}\right)\left(t_{j-1}+t_{j}+t_{j+1}\right) .
$$

Thus we have

$$
\begin{aligned}
\frac{1}{6} \sum_{j=1}^{N} M_{j}\left(h_{j}+h_{j+1}\right)\left(t_{j-1}+t_{j}+t_{j+1}\right) & =\sum_{j=1}^{N} t_{j}\left(\frac{f_{j+1}-f_{j}}{h_{j+1}}-\frac{f_{j}-f_{j-1}}{h_{j}}\right) \\
& =\sum_{j=1}^{N}\left(f_{j}-f_{j-1}\right),
\end{aligned}
$$

which is equal to 0 . Thus (36) becomes

$$
S_{\Delta}(z)=\frac{1}{2 \pi i} \sum_{j=1}^{N} q_{\Delta, j}(z) \log \frac{t_{j}-z}{t_{j-1}-z} .
$$

The unit circle. The inverse of the coefficient matrix for (3) or (7) when the curve $K$ is the unit circle and the intervals $t_{k-1}, t_{k}$ are of equal length can be easily obtained. We have $t_{k-1}=e^{i k \alpha}(\alpha=2 \pi / N)$ and

$$
\lambda_{k}=\frac{t_{k+1}-t_{k}}{t_{k+1}-t_{k-1}}=\frac{e^{i \alpha}}{e^{i \alpha}+1}=\lambda
$$

The coefficient matrix for (3) in this situation is the $N \times N$ circulant

$$
\begin{gathered}
C[2, \lambda, 0, \ldots, 0,1-\lambda], \\
{\left[\begin{array}{ccccc}
2 & \lambda & 0 & \ldots & 1-\lambda \\
1-\lambda & 2 & \lambda & \ldots & 0 \\
0 & 1-\lambda & 2 & \ldots & 0 \\
\vdots & & & & \\
\lambda & 0 & 0 & \ldots & 2
\end{array}\right] .}
\end{gathered}
$$

Representing the inverse matrix by the circulant $C\left[a_{1}^{-1}, a_{2}^{-1}, \ldots, a_{N}^{-1}\right]$, we have

$$
\begin{gathered}
2 a_{1}^{-1}+\lambda a_{N}^{-1}+(1-\lambda) a_{2}^{-1}=1 \\
2 a_{2}^{-1}+\lambda a_{1}^{-1}+(1-\lambda) a_{3}^{-1}=0 \\
\vdots \\
2 a_{N}^{-1}+\lambda a_{N-1}^{-1}+(1-\lambda) a_{1}^{-1}=0 .
\end{gathered}
$$


Thus the quantities $a_{k}^{-1}$ satisfy the difference equation $\lambda a_{k-1}^{-1}+2 a_{k}^{-1}+(1-\lambda) a_{k+1}^{-1}=0$ $(k=2,3, \ldots, N-1)$, subject to the two conditions represented by the first and last of equations (38).

The roots of the characteristic equation are

$$
\left(-1 \pm(1-\lambda(1-\lambda))^{1 / 2}\right) / \lambda=e^{-i \alpha / 2}\left[-2 \cos \alpha / 2 \pm\left(4 \cos ^{2} \alpha / 2-1\right)^{1 / 2}\right] .
$$

Here the radicand is positive if $\alpha<2 \pi / 3$; i.e., if $N>3$. Designate these roots by $r$ and $s$. Then

and

$$
a_{k}^{-1}=A r^{k}+B s^{k},
$$

$$
\begin{aligned}
A\left[\lambda r^{N-1}+2 r^{N}+(1-\lambda) r\right]+B\left[\lambda s^{N-1}+2 s^{N}+(1-\lambda) s\right] & =0, \\
A\left[\lambda r^{N}+2 r+(1-\lambda) r^{2}\right]+B\left[\lambda s^{N}+2 s+(1-\lambda) s^{2}\right] & =1 .
\end{aligned}
$$

Hence

$$
A=\frac{-s}{\lambda(r-s)\left(r^{N}-1\right)}, \quad B=\frac{r}{\lambda(r-s)\left(s^{N}-1\right)},
$$

and, since $r s=\lambda /(1-\lambda)=e^{i \alpha}$,

$$
a_{k}^{-1}=-\frac{\cos \alpha / 2}{\left(4 \cos ^{2} \alpha / 2-1\right)^{1 / 2}} \cdot \frac{r^{N-k+1}+r^{k-1}}{r^{N}-1} .
$$

Alternative type of complex spline. Closely related to the same problem of approximating a function analytic interior to the curve $K$ are the complex-valued splines of the real variable $s$ (arc length) on $K$. These have been investigated to a limited extent previously [24]. Here we can sharpen and extend the earlier results.

Equations (3) become

$$
\mu_{j} \tilde{M}_{j-1}+2 \tilde{M}_{j}+\lambda_{j} \tilde{M}_{j+1}=6 f\left[s_{j-1}, s_{j}, s_{j+1}\right],
$$

with $\tilde{M}_{j}=d^{2} f / d s^{2}$, but now the quantities $h_{j}=s_{j}-s_{j-1}$ and $\mu_{j}=1-\lambda_{j}=h_{j} /\left(h_{j}+h_{j+1}\right)$ are real and positive. The coefficient matrix has dominant main diagonal for all meshes on $K$ and the inverse matrix has row-max norm not greater than unity. Similarly, equations (7) become

$$
\lambda_{j} \tilde{m}_{j-1}+2 \tilde{m}_{j}+\mu_{j} \tilde{m}_{j+1}=3\left(\mu_{j} f\left[s_{j}, s_{j+1}\right]+\lambda_{j} f\left[s_{j-1}, s_{j}\right]\right)
$$

with $\tilde{m}_{j}=d f / d s$.

The complex spline within the region $R$ is defined as

$$
\tilde{S}_{\Delta}(z)=\frac{1}{2 \pi i} \int_{K} \frac{\tilde{q}_{\Delta}(s)}{t(s)-z} d t(s),
$$

where $t=t(s)$ is the complex representation of the curve $K$ and $\tilde{q}_{\Delta}(s)$ is the complexvalued spline given in the interval $s_{j-1} \leqq s \leqq s_{j}$ by

$$
\begin{aligned}
\tilde{q}_{\Delta}(s)= & \frac{\tilde{M}_{j-1}}{6 h_{j}}\left(s_{j}-s\right)^{3}+\frac{\tilde{M}_{j}}{6 h_{j}}\left(s-s_{j-1}\right)^{3} \\
& +\left(f_{j-1} / h_{j}-M_{j-1} h_{j} / 6\right)\left(s_{j}-s\right)+\left(f_{j} / h_{j}-M_{j} h_{j} / 6\right)\left(s-s_{j-1}\right),
\end{aligned}
$$


or, equivalently, by

$$
\begin{aligned}
q_{\Delta}(s)= & \frac{\tilde{m}_{j-1}}{h_{j}^{2}}\left(s_{j}-s\right)^{2}\left(s-s_{j-1}\right)+\frac{\tilde{m}_{j}}{h_{j}^{2}}\left(s-s_{j-1}\right)^{2}\left(s_{j}-s\right) \\
& +\frac{f_{j-1}}{h_{j}^{3}}\left(s_{j}-s\right)^{2}\left[2\left(s-s_{j-1}\right)+h_{j}\right]+\frac{f_{j}}{h_{j}^{3}}\left(s-s_{j-1}\right)^{2}\left[2\left(s_{j}-s\right)+h_{j}\right] .
\end{aligned}
$$

As $z$ in $R$ approaches $t(s)$ on $K, \widetilde{S}_{\Delta}(z)$ approaches

$$
\tilde{S}_{\Delta}(t)=\frac{1}{2} q_{\Delta}(s)+\frac{1}{2 \pi i} \int_{K} \frac{\tilde{q}_{\Delta}(s)}{\tau(s)-t} d \tau(s) .
$$

Similarly $\tilde{S}_{\Delta}^{\prime}(z)$ and $\tilde{S}_{\Delta}^{\prime \prime}(z)$ approach, respectively,

$$
\tilde{S}_{\Delta}^{\prime}(t)=\frac{1}{2} \frac{d \tilde{q}_{\Delta}}{d s} \cdot \frac{d s}{d t}+\frac{1}{2 \pi i} \int_{K} \frac{\left(d \tilde{q}_{\Delta} / d s\right) \cdot(d s / d \tau)}{\tau(s)-t} d \tau(s),
$$

and

$$
S_{\Delta}^{\prime \prime}(t)=\frac{1}{2}\left[\frac{d^{2} \tilde{q}_{\Delta}}{d s^{2}}\left(\frac{d s}{d t}\right)^{2}+\frac{d \tilde{q}_{\Delta}}{d s} \cdot \frac{d^{2} s}{d t^{2}}\right]+\frac{1}{2 \pi i} \int_{K} \frac{\frac{d^{2} \tilde{q}_{\Delta}}{d s^{2}}\left(\frac{d s}{d \tau}\right)^{2}+\frac{d \tilde{q}_{\Delta}}{d s} \cdot \frac{d^{2} s}{d \tau^{2}}}{\tau(s)-t} d \tau(s) .
$$

The properties corresponding to Theorems 1-6 and their corollaries carry over to the present situation. Some simplification in the proofs results from the fact that we are concerned here with splines in the real variable $s$. The convergence properties set forth in Theorem 7-10 are valid in this situation and follow as in the preceding section.

The cubic splines in the real variable $s$ have the important property that they satisfy two fundamental integral relations. We have

$$
\int_{K}\left|\frac{d^{2} f}{d s^{2}}\right|^{2} d s=\int_{K}\left|\frac{d^{2} f}{d s^{2}}-\frac{d^{2} \tilde{q}_{\Delta}}{d s^{2}}\right|^{2} d s+\int_{K}\left|\frac{d^{2} \tilde{q}_{\Delta}}{d s^{2}}\right|^{2} d s+2 \mathscr{R} \int_{K}\left(\frac{d^{2} f}{d s^{2}}-\frac{d^{2} \tilde{q}_{\Delta}}{d s^{2}}\right)\left(\overline{\frac{d^{2} \tilde{q}_{\Delta}}{d s^{2}}}\right) d s
$$

and integration by parts gives

$$
\int_{K}\left(\frac{d^{2} f}{d s^{2}}-\frac{d^{2} \tilde{q}_{\Delta}}{d s^{2}}\right)\left(\overline{\frac{d^{2} q}{d s^{2}}}\right) d s=\left.\sum_{j=1}^{N}\left(\frac{d f}{d s}-\frac{d \tilde{q}_{\Delta}}{d s}\right)\left(\overline{\frac{d^{2} \tilde{q}_{\Delta}}{d s^{2}}}\right)\right|_{t_{j-1}} ^{t_{j}}-\left.\sum_{j=1}^{N}\left(f-\tilde{q}_{\Delta}\right)\left(\overline{\frac{d^{3} q}{d s^{3}}}\right)\right|_{t_{j-1}} ^{t_{j}}=0 .
$$

The First Integral Identity which results since $\tilde{q}_{\Delta}$ is a spline of interpolation to $f$ on $\Delta$ is

$$
\int_{K}\left|\frac{d^{2} f}{d s^{2}}\right|^{2} d s=\int_{K}\left|\frac{d^{2} f}{d s^{2}}-\frac{d^{2} \tilde{q}_{\Delta}}{d s^{2}}\right|^{2} d s+\int_{K}\left|\frac{d^{2} \tilde{q}_{\Delta}}{d s^{2}}\right|^{2} d s
$$

Actually we require only that $d f / d s$ be absolutely continuous on $K$ and that $d^{2} f / d s^{2}$ be Lebesque square integrable.

As an immediate consequence we have [24] the following extremal properties:

(i) The quantity $\int_{K}\left|d^{2}\left(f-h_{\Delta}\right) / d s^{2}\right|^{2} d s$ for arbitrary cubic splines $h_{\Delta}(s)$ on $\Delta$ is minimum when $h_{\Delta}(s)=\tilde{q}_{\Delta}(s)$. 
(ii) Of all the $C^{2}$ functions $g(s)$ on interpolating to $f(s)$ on $\Delta$, the quantity $\int_{K}\left|q^{\prime \prime}(s)\right|^{2} d s$ is minimum when $g(s)=\tilde{q}_{\Delta}(s)$.

We define a pseudo inner product

$$
(u, v)=\int_{K} u^{\prime \prime}(s) \overline{v^{\prime \prime}(s)} d s
$$

when $u(s)$ and $v(s)$ are absolutely continuous on $K$ (necessarily periodic in $s$ ) with $L^{2}$-integrable second derivatives. We say that $u$ and $v$ are orthogonal if $(u, v)=0$. An immediate consequence [21] is that if $\Delta$ and $\Delta^{\prime}$ are two meshes on $K$ with $\Delta \subset \Delta^{\prime}$, if $h_{\Delta}$ and $h_{\Delta^{\prime}}$ are splines on $\Delta$ and $\Delta^{\prime}$ such that $h_{\Delta^{\prime}}=0$ on $\Delta$, then $h_{\Delta}$ and $h_{\Delta^{\prime}}$ are orthogonal.

Using a sequence of imbedded meshes $\left\{\Delta_{k}\right\}$ with $\left\|\Delta_{k}\right\| \rightarrow 0$ and with $\Delta_{k+1}$ consisting of the points of $\Delta_{k}$ together with a point $s_{k+1}$ not in $\Delta_{k}$, and taking $h_{\Delta_{k+1}}$ to be the complex cubic spline on $\Delta_{k+1}$ which is 1 at $s_{k+1}$ and 0 on $\Delta_{k}$, we obtain a complete sequence of orthogonal splines which is a dense subset of the space of continuous functions on $K$. Many other orthogonal sequences may be constructed, of course.

We obtain further that if $\left\{\Delta_{k}\right\}$ is any sequence of subdivisions of $K$ with $\left\|\Delta_{k}\right\| \rightarrow 0$ as $k \rightarrow \infty$, and if $f^{\prime}(s)$ is absolutely continuous with $f^{\prime \prime}(s) L^{2}$-integrable, with $q_{\Delta_{k}}(s)$ the corresponding cubic splines on $K$ of interpolation to $f(s)$ on $\Delta_{k}$, then $q_{\Delta_{k}}^{\prime \prime}(s)$ converges in the mean to $f^{\prime \prime}(s)$ :

$$
\lim _{k \rightarrow \infty} \int_{K}\left|\left(f-q_{\Delta_{k}}\right)^{\prime \prime}\right|^{2} d s=0
$$

The set of all functions $f(s)$ with absolutely continuous first derivatives and $L^{2}$ integrable second derivatives form a Hilbert space with respect to the pseudoscalar product (41). A basis for this space may be taken, for example, to be the sequence of orthogonal splines introduced above.

We note for the sake of completeness the so-called second integral relation [23], valid if $f(s)$ is Lebesgue integrable and $q_{\Delta}(s)$ is the complex cubic spline of interpolation to $f(s)$ on $K$ :

$$
\int_{K}\left|\left(f-q_{\Delta}\right)^{\prime \prime}\right|^{2} d s=\int_{K}\left(f-q_{\Delta}\right) \overline{f^{1 \mathrm{v}}}(s) d s
$$

\section{REFERENCES}

1. I. J. Schoenberg, Contributions to the problem of approximation of equidistant data by analytic functions, Quart. Appl. Math. 4 (1946), 45-99, 112-141.

2. I. J. Schoenberg and Anne Whitney, On Pólya frequency functions. III. The positivity of translation determinants with application to the interpolation problem by spline curves, Trans. Amer. Math. Soc. 74 (1953), 246-259.

3. J. C. Holladay, Smoothest curve approximation, Math. Comp. 11 (1957), 233-243. 
4. I. J. Schoenberg, Spline functions, convex curves, and mechanical quadrature, Bull. Amer. Math. Soc. 64 (1958), 352-357.

5. R. S. Johnson, On monosplines of least deviation, Trans. Amer. Math. Soc. 96 (1960), 458-477.

6. G. Birkhoff and H. Garabedian, Smooth surface interpolation, J. Math. Phys. 39 (1960), 258-268.

7. F. Theilheimer and W. Starkweather, The fairing of ship lines on a high-speed computer, Math. Comp. 15 (1961), 338-355.

8. J. L. Walsh, J. H. Ahlberg and E. N. Nilson, Best approximation properties of the spline fit, J. Math. Mech. 11 (1962), 225-234.

9. C. deBoor, Bicubic spline interpolation, J. Math. Phys. 41 (1962), 212-218.

10. B. Asker, The spline curve, a smooth interpolating function used in numerical design of ship-lines, Nordisk Tidskr. Informations-Behandling 2 (1962), 76-82.

11. F. Landis and E. N. Nilson, "The determination of therodynamic properties by direct differentiation techniques," Progress in International Research on Thermodynamics and Transport Properties, Amer. Soc. of Mech. Engineers, 1962.

12. J. H. Ahlberg and E. N. Nilson, Convergence properties of the spline fit, J. Soc. Indust. Appl. Math. 11 (1963), 95-104.

13. C. deBoor, Best approximation properties of spline functions of odd degree, J. Math. Mech. 12 (1963), 747-749.

14. I. J. Schoenberg, Spline interpolation and the higher derivatives, Proc. Nat. Acad. Sci. U.S.A. 51 (1964), 24-28.

15. - Spline interpolation and best quadrature formulae, Bull. Amer. Math. Soc. 70 (1964), 143-148.

16. - On trigonometric spline interpolation, J. Math. Mech. 13 (1964), 795-826.

17. G. Birkhoff and C. deBoor, Error bounds for spline interpolation, J. Math. Mech. 13 (1964), 827-836.

18. T. N. E. Greville, Numerical procedures for interpolation by spline functions, J. SIAM Ser. B Numer. Anal. 1 (1964), 53-68.

19. — Interpolation by generalized splines, MRC Rep. 476, Univ. of Wisconsin, Madison, 1964.

20. I. J. Schoenberg, On best approximation of linear operators, Nederl. Akad. Wetensch. Proc. Ser. A 67=Indag. Math. 26 (1964), 155-163.

21. J. H. Ahlberg, E. N. Nilson and J. L. Walsh, Fundamental properties of generalized splines, Proc. Nat. Acad. Sci. U.S.A. 52 (1964), 1412-1419.

22. - Best approximation properties of higher-order spline approximations, J. Math. Mech. 14 (1965), 231-244.

23. - Convergence properties of generalized splines, Proc. Nat. Acad. Sci. U.S.A. 54 (1965), 344-350.

24. J. H. Ahlberg and E. N. Nilson, Orthogonality properties of spline functions, J. Math. Anal. Appl. 11 (1965), 321-337.

25. J. H. Ahlberg, E. N. Nilson and J. L. Walsh, Extremal, orthogonality, and convergence properties of multidimensional splines, J. Math. Anal. Appl. 11 (1965), 27-48.

26. J. H. Ahlberg and E. N. Nilson, Solution of differential equations by the method of cardinal splines, J. SIAM Numer. Anal. 3 (1966), 173-182, Walsh Jubilee Volume.

27. F. B. Hildebrand, Introduction to numerical analysis, McGraw-Hill, New York, 1956, p. 38.

28. A. Sharma and A. Meir, Convergence of spline functions, Abstract 64T-496, Notices Amer. Math. Soc. 11 (1964), 768. 
29. N. I. Muskhelishvili, Some basic problems of the mathematical theory of elasticity, Noordhoff, Groningen, 1953, pp. 57ff.

30. J. L. Walsh, Interpolation and approximation by rational functions in the complex domain, Colloq. Publ. Vol. 20, Amer. Math. Soc., Providence, R. I., 1935, p. 10.

United Aircraft Research Laboratories,

EAST HARTFORD, CONNECTICUT

Pratt \& Whitney Aircraft,

EAST HARTFORD, CONNECTICUT

UNIVERSITY OF MARYLAND,

College Park, Maryland 